\title{
Fundamentals and Applications of Inertial Microfluidics: A Review
}

\author{
Jun Zhang, Sheng Yan ${ }^{\mathrm{a}}$, Dan Yuan ${ }^{\mathrm{a}}$, Gursel Alici ${ }^{\mathrm{a}}$, Nam-Trung Nguyen ${ }^{\mathrm{b}}$, Majid Ebrahimi \\ Warkiani ${ }^{\mathrm{c}}$, Weihua $\mathrm{Li}^{\mathrm{a}^{*}}$ \\ ${ }^{\text {a }}$ School of Mechanical, Materials and Mechatronic Engineering, University of Wollongong, \\ Wollongong, NSW 2522, Australia \\ ${ }^{\mathrm{b}}$ Queensland Micro- and Nanotechnology Centre, Griffith University, Brisbane QLD 4111, \\ Australia \\ c School of Mechanical and Manufacturing Engineering, Australian Centre for NanoMedicine, \\ University of New South Wales, Sydney, NSW 2052, Australia
}

\begin{abstract}
:
In the last decade, inertial microfluidics has attracted significant attention and a wide variety of channel designs that focus, concentrate and separate particles and fluids have been demonstrated. In contrast to conventional microfluidic technologies, where fluid inertia is negligible and flow remains almost within Stokes flow region with very low Reynolds number $\operatorname{Re}<<1$, inertial microfluidics works in the intermediate Reynolds number range $(\sim 1$ $<\operatorname{Re}<\sim 100$ ) between Stokes and turbulent regimes. In this intermediate range, both inertia and fluid viscosity are finite, and bring about several intriguing effects that form the basis of inertial microfluidics including: (i) inertial migration and (ii) secondary flow. Due to the superior features of high-throughput, simplicity, precise manipulation and low cost, inertial microfluidics is a very promising candidate for cellular sample processing, especially for sample with low abundant targets. In this review, we first discuss the fundamental kinematics of particles in microchannels to familiarise readers with mechanisms and underlying physics in inertial microfluidic systems. We then present a comprehensive review of recent developments and key applications of inertial microfluidic systems according to their microchannel structures. Finally, we discuss the perspective of employing fluid inertia in microfluidics for particle manipulation. Due to the superior benefits of inertial microfluidics, this promising technology will still be an attractive topic in the near future, with more novel designs and further applications in biology, medicine and industry on the horizon.
\end{abstract}

\section{INTRODUCTION}

Microfluidics is referred to the science that deals with the behaviour, precise control and manipulation of fluids and particles in the scale of tens to hundreds of micrometres ${ }^{1}$. Microfluidics is also considered as a platform for biomedical and chemical applications, called Lab-on-a-Chip (LOC), or micro Total Analysis Systems ( $\mu$ TAS) ${ }^{2}$. Benefiting from the precise control and manipulation of biological particles and its surrounding microenvironment, this fascinating technology holds superior advantages compared to conventional macro-scale platforms (e.g. centrifuge, and flow cytometry etc.). Microfluidics 
benefits include but is not limited to (i) reduced sample and reagent volumes, (ii) fast sample processing, (iii) high sensitivity, (iv) low cost, (v) improved portability, and (vi) the potential to be highly integrated and automated to reduce human intervention and error ${ }^{3}$.

Precise manipulation such as focusing, separation and fractionation of bio-particles is an indispensable capability of microfluidics. For instance, three-dimensional focusing and ordering of micro-particles along a single line allow for single-cell level detection and analysis, which is an important component of on-chip flow cytometry. Furthermore, separation of micro-particles/cells according to their unique biophysical signatures such as size, density, shape, deformability, and surface proteins allows for a wide range of applications such as preparation of biological sample (e.g., blood, lymphatic tissue and tissue digestates etc.), isolation and enrichment of target cells as well as water purification ${ }^{4,5}$.

A number of technologies have already been proposed and developed to manipulate particles in microfluidic systems. According to the source of the manipulating forces, these technologies can be categorised as active and passive types. Active technologies such as dielectrophoresis (DEP) ${ }^{6}$, magnetophoresis (MP) ${ }^{7}$, acoustophoresis (AP) ${ }^{8}$ and optical tweezer ${ }^{9}$ rely on external force fields, whereas passive technologies depend entirely on the channel geometry or intrinsic hydrodynamic forces, such as pinched flow fractionation (PFF) ${ }^{10}$, deterministic lateral displacement (DLD) ${ }^{11}$ and inertial microfluidics ${ }^{4}$. An active technology generally allows for a more precise control of target particles, as well as being tuneable in real time. However, the flow speed is always limited because the external force field should overcome the hydrodynamic drag to achieve effective functionality. In contrast, a passive microfluidic technology is always very simple, robust and works with a relatively higher flow rate.

Among passive microfluidic technologies, inertial microfluidics has recently attracted a great attention due to its precise manipulation, simple structure and high throughput. In contrast to the common sense that, in microfluidics, fluid inertia is negligible (Stokes flow regime, $\mathrm{Re} \rightarrow$ 0 , $\operatorname{Re}=\rho U H / \mu$, where $\rho, U$ and $\mu$ are fluid density, average velocity and dynamic viscosity respectively, and $H$ is characteristic channel dimension), inertial microfluidics works in an intermediate range $(\sim 1<\operatorname{Re}<\sim 100)$ between Stokes regime and turbulent regime $(\operatorname{Re} \sim$ 2000), where both inertia and viscosity of the fluid are finite. The finite inertia of the fluid brings about several intriguing inertial effects that form the basis of inertial microfluidics including: (i) inertial migration and (ii) secondary flow.

Inertial migration is a phenomenon where randomly dispersed particles in the entrance of a straight channel migrate laterally to several equilibrium positions after a long enough distance, which was first observed more than 50 years ago ${ }^{12,13}$. After the first observation of inertial migration, a number of experimental studies and theoretical analyses were conducted to explore the underlying mechanism of this phenomenon ${ }^{14-21}$. This phenomenon had not found its practical application until the emergence of microfluidic technology, where particle size is comparable with characteristic dimension of microchannel. The inertial migration phenomenon has been widely recognised by the counteraction of two inertial effects: 1) the shear gradient lift force $F_{L S}$, due to the curvature of the fluid velocity profile and its interaction with a particle, which directs particles away from channel centre, and 2) the wall 
lift force $F_{L W}$, a result of the flow field interaction between the suspending particle and the adjacent walls, which repels the particle away from the wall. The inertial equilibrium positions of particles result from the balance between these two inertial lift forces, and they are normally around 0.6 times of channel radius to the channel centreline in a circular channel 13,22 .

The secondary flow usually appears in a curved channel ${ }^{23}$ or a straight channel with disturbance obstacles ${ }^{24-26}$. In a curved channel, the secondary flow is induced by a pressure gradient in the radial direction, because of fluid momentum mismatch in the centre and nearwall region within the curvature ${ }^{4}$. The fluid elements near the channel centreline have a higher momentum than that near the wall, therefore flow outwards, and drive relative stagnant fluid elements near the channel wall inwards along the circumference, forming two counter-rotating streams, called Dean vortex. Introducing this secondary flow in inertial focusing brings several benefits. For example, Dean vortex can modify the inertial equilibrium positions by imposing an additional viscous drag force on particles perpendicular to the main stream. Size-dependent differential focusing of particles according to the ratio of inertial lift force and secondary flow drag $\left(F_{L} / F_{D}\right)$ promises the complete particle separation. In addition, Dean vortex could reduce the channel length/footprint, due to the mixing effects of secondary flow, assisting particles to reach equilibrium position more quickly ${ }^{4}$.

In this review, we will first discuss the fundamental kinematics of micro-particles inside microchannels. We then present a comprehensive review of the recent progress of inertial microfluidics according to the structure of functional microchannels, because the structure of microchannel is the most crucial parameter that determines the functionality and performance of inertial microfluidic devices. Finally, the limitation of current investigations will be discussed. We then propose several future directions of inertial microfluidics. We hope that this review could serve as a comprehensive guide for readers who are new to the area of inertial microfluidics. For readers with a decent background, we hope that the review can provide extended understanding of fundamental mechanism, inspire novel device design or initiate expanded application of inertial microfluidics.

\section{FUNDAMENTAL DYNAMICS OF PARTICLE MOVEMENT IN A MICROFLUIDIC CHANNEL}

\subsection{Viscous drag force}

Drag force arises when an object moves through a fluid or, relatively, when the fluid flows past an object. The origin of the drag force lies in the need to displace the elements of the fluid out of the way of the moving object. The drag force on a moving spherical particle can be expressed as:

$$
F_{\text {drag }}=S * f_{\text {drag }}=\pi a^{2} f_{\text {drag }} / 4
$$

where $S$ is the cross-sectional area of moving particles, and $a$ is the diameter of particle. $f_{\text {drag }}$ is viscous drag coefficient which is always determined by the particle Reynolds number $R e^{\prime}=v_{t} \rho_{f} a / \mu$, here $v_{t}$ is relative velocity of fluid to particle ${ }^{27}$. 
The viscous drag coefficient $f_{\text {drag }}$ can be divided into four sections according to the range of particle Reynolds number $R e^{\prime 27}$ :

(a) When $10^{-4}<R e^{\prime}<0.2$

$$
f_{\text {drag }}=12 \frac{\mu v_{t}}{a}
$$

Then

$$
F_{d r a g}=3 \pi \mu a v_{t}
$$

This is actually the well-known formula of the Stokes drag, which has been widely used for analytical analysis because of its simplicity, especially when relative velocity of fluid to particle $v_{t}$ is very small.

(b) If $0.2<R e^{\prime}<500 \sim 1000$

$$
f_{\text {drag }}=12 \frac{\mu v_{t}}{a}\left(1+0.15 R e^{\prime 0.687}\right)
$$

then

$$
F_{\text {drag }}=3 \pi \mu a v_{t}\left(1+0.15 R e^{\prime 0.687}\right)
$$

(c) If $500 \sim 1000<R e^{\prime}<2 \times 10^{5}$

$$
f_{\text {drag }}=0.22 \rho_{f} v_{t}^{2}
$$

then

$$
F_{\text {drag }}=0.055 \pi a^{2} \rho_{f} v_{t}^{2}
$$

Subsequently, Khan and Richardson examined the experimental data and suggested an equation that suits for values of $R e^{\prime}$ up to $10^{527}$ :

$$
\begin{gathered}
f_{\text {drag }}=\left(1.84 R e^{\prime-0.31}+0.293 R e^{\prime 0.06}\right)^{3.45} \rho_{f} v_{t}^{2} \\
F_{\text {drag }}=\frac{\pi}{4} a^{2} \rho_{f} v_{t}^{2}\left(1.84 R e^{\prime-0.31}+0.293 R e^{\prime 0.06}\right)^{3.45}
\end{gathered}
$$

In inertial microfluidics, viscous drag force is comprised of two components: along 1) mainstream direction, due to the axial velocity difference between fluid and the suspended particles; 2) cross-section, due to the secondary flow induced by the channel curvature, or disturbance structures.

\subsection{Diffusion}

Brownian motion is the random motion of suspending particles immersed in a fluid (liquid or gas), which is resulted from their frequent collision with the quick atoms or molecules in the gas or liquid. Diffusion is ubiquitous in particle-fluid system. According to EinsteinSmoluchowski theory, the mean square distance that a particle diffuses in time $t$ is expressed as ${ }^{28}$ :

$$
<r^{2}>=6 D t
$$


where $D$ is the diffusion coefficient. According to the Stokes-Einstein relationship, the diffusion coefficient is given as:

$$
D=\frac{k T}{3 \pi \mu a}
$$

Where $k$ is the Boltzmann's constant, which is about $1.3806488 \times 10^{-23} \mathrm{~J} / \mathrm{K} . T$ is the absolute temperature ${ }^{28}$. From the above equation, we can see that the higher the temperature $T$, the larger is the diffusion coefficient $D$. In contrast, the larger the particle size $a$ or the higher the medium viscosity $\mu$, the smaller is the diffusion coefficient. When the particle-fluid system is not static, but moving along the microchannel, the Péclet number can be employed to evaluate the relative importance of convection to diffusion ${ }^{29}$ :

$$
P e=\frac{L U}{D}
$$

where $L$ is characteristic dimension of channel and $U$ is characteristic velocity of fluid. The large Péclet number indicates that convection dominates the transport of particles in the flow, while a small Péclet number means that diffusion plays a primary role in transport. In inertial microfluidics, diffusion is normally neglected in the analysis of microscale particle movement in inertial microfluidics.

\subsection{Magnus force--rotation-induced lift force}

In an in-viscous flow with uniform velocity $U$, a stationary cylinder is rotating with constant angular velocity $\Omega$, see figure 1(a). Assuming no slip velocity at the surface of the cylinder, the fluid velocity at the bottom part is lower than the velocity at the upper part. According to the Bernoulli principle, the pressure is higher at the lower part than that in the upper part of the cylinder. As a result, a lift force $F_{L R}$ is developed to lift the cylinder. The magnitude of this lift force, per unit length of the cylinder is ${ }^{30}$ :

$$
\vec{F}_{L R}=\pi \rho_{f}^{2} a \vec{U} \times \vec{\Omega}
$$

Similarly for a rigid sphere rotating in a fluid, a lateral lift force appears due to the transverse pressure difference, and this force is called "Magnus lift force" ${ }^{31}$ :

$$
\vec{F}_{L R}=\frac{1}{8} \pi a^{3} \rho_{f} \vec{U} \times \vec{\Omega}
$$

If the sphere is not stationary, but simultaneously translating through the fluid with a velocity $\vec{u}_{p}$, replacing the fluid velocity vector $\vec{U}$ by relative velocity $\left(\vec{u}_{f}-\vec{u}_{p}\right)$ results in:

$$
\vec{F}_{L R}=\frac{1}{8} \pi d^{3} \rho_{f}\left(\vec{u}_{f}-\vec{u}_{p}\right) \times \vec{\Omega}
$$

The direction of Magnus force is perpendicular to the plane defined by the vectors of the relative velocity and the axis of rotation. In the case of a sphere rotating with an angular velocity $\vec{\Omega}_{\mathrm{s}}$ in a rotational flow field, the vector $\vec{\Omega}$ represents the relative rotation between the fluid and the sphere:

$$
\vec{\Omega}=\vec{\Omega}_{\mathrm{s}}-0.5 \nabla \times \vec{u}_{f}
$$


Magnus force can be considered as a result of the pressure difference induced by the streamline asymmetry, due to the rotation of the sphere. Although above particular expressions are only valid for low Reynolds numbers, this kind of lift force due to the rotation of a body is also present in a more strongly inertial flow ${ }^{32}$. In fact, as a result of the outer velocity field disturbance by the rotation, where fluid inertia is essential, the Magnus force would not appear in the creeping flow, but present in all rotating flows with finite relative velocity no matter it is viscous or inviscid ${ }^{30}$.

a

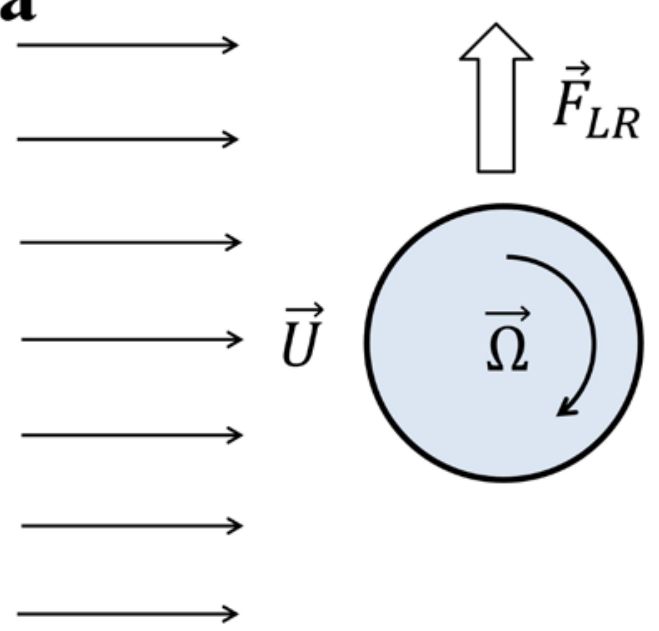

b
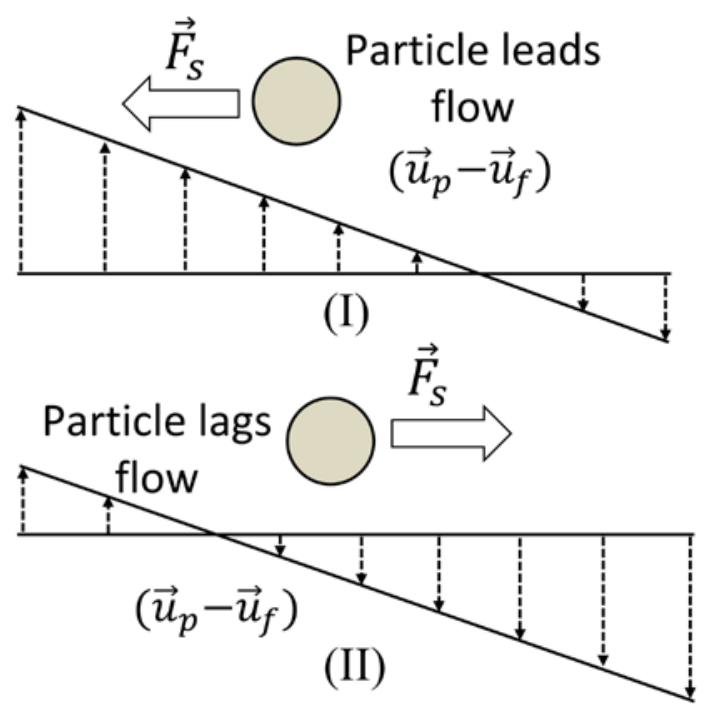

(II)

d

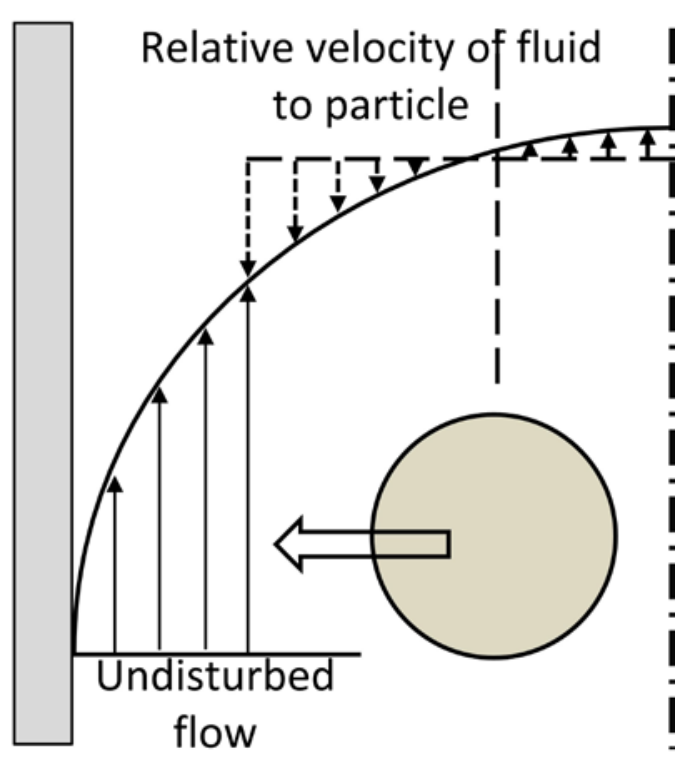

Figure 1 (a) Rotation induced lift force for a rigid cylinder or sphere in a uniform flow. (b) Saffman force on a sphere due to slip-shear motion in a simple shear flow. The direction of lateral lift force always directs toward the side where magnitude of relative velocity is maximum. (c) Boundaries retard the motion of the particle no matter whether the main flow direction is parallel (I) or perpendicular (II) to the wall boundary. An additional wall lift force directs perpendicularly to the main flow direction when particles are moving parallel to the boundary. (d) Shear gradient lift force on a particle in a Poiseuille flow. In the moving frame with the particle, 
the relative velocity of fluid to particle is larger on the wall side due to the parabolic fluid velocity profile. Therefore, shear-gradient lift force directs toward the near-by wall ${ }^{33}$.

\subsection{Saffman force--slip-shear induced lift force}

Inertial migration is an interesting phenomenon where randomly dispersed particles in the entrance of a straight micro-channel migrate laterally to a narrow annulus at about 0.6 times of the tube radii from the axis, which was first quantitatively measured by Segre and Silberberg in 1960s ${ }^{12,13}$. After that, a series of analytical analysis and numerical simulation have been conducted to explain this counter-intuitive phenomenon ${ }^{14-19}$. However, the full problem is more complex, as not only the effect of inertia needs to be calculated for a particle in a parabolic velocity profile, but also the presence of the tube walls must be taken into account. The walls are clearly very important to the existence of the phenomenon. Without channel walls, the flow will be uniform and there is even no velocity gradient, generating no rotational motion on a force-free rigid sphere, and consequently no lateral lift force appears. The wall effects at least act in two different ways: First, the existence of walls creates a fluid velocity gradient (shear rate), and corresponding shear-induced particles rotation. The extra drag caused by walls makes the particle lagging behind the fluid. This slip-shear motion will generate a lateral force on the particles called the Saffman force. Second, the flow field around the particle is disturbed by the presence of the walls and the inertial effects will differ from those for a particle in an unbounded flow, especially when the particle is close to the walls ${ }^{34}$. Here, we focus on the first effect and only consider the force on a sphere in an unbounded simple shear flow, i.e. constant shear rate and zero shear gradient. The second effect of the wall will be detailed in the section of "Wall-induced lift force". For Poiseuille flow with non-zero shear gradient, the effects of parabolic velocity profile on particle migration will be discussed in the section of "Shear-gradient lift force".

Using the matched asymptotic expansion method, Saffman ${ }^{34}$ calculated the lateral lift force on a sphere in a simple unbounded shear flow. The force arises from the interaction of the Stokeslet velocity field of the particle and the velocity gradient of the bulk flow. The magnitude of this force is:

$$
F_{S}=\frac{K}{4} V a^{2}\left(\gamma v^{-1}\right)^{1 / 2}
$$

where $K$ is a numerical constant $(K \sim 81.2), \gamma$ is velocity gradient (or shear rate), $v$ is the kinetic viscosity and $V$ is the relative velocity between particle and fluid at the streamline through the centre of the particle. It should be pointed out that this force is the only effect of shear rate, independent of the rotation of particle. In the real situation, the spheres are free to move, and a relative rotation will be induced by the shear. Consequently, a Magnus-type force may appear on the sphere. In most practical flows, the effects of rotation and shear cannot be considered as independent, and a simple superposition of the two lift forces would inevitably bring errors ${ }^{30}$. Unless the rotation speed is much greater than the rate of shear, for a freely rotating particle $\Omega=\frac{1}{2} \gamma$, Saffman force $F_{S}$, caused by the interaction of slip velocity (Stokeslet flow field) and shear, will generally be at least one order of magnitude larger than Magnus force $F_{L R}$ caused by the interaction of slip and particle rotation at low Reynolds numbers ${ }^{32}$. 
The direction of Saffman force is always towards the side where the magnitude of relative velocity $V$ is maximum, Figure 1(b). If the particles are leading the flow, Saffman force directs to the stagnant wall in a simple shear flow; if the particles are lagging the flow, Saffman force points to the moving wall. In the case of a neutrally buoyant particles in a Poiseuille flow, the particle is force-free and the Stokeslet generated by the lag of the particle relative to the shear flow (slip-shear) is balanced by an opposite Stokeslet originating from the curvature of the base flow, so there is basically no net Stokeslet in the base flow, consequently no Saffman force acts on the sphere ${ }^{32}$. Saffman force is more relevant in the case of non-neutrally buoyant particles in a vertical flow, or additional outer force (electrical or magnetic) ${ }^{35,36}$ acting on the particle to lag or lead fluid flow, creating obvious net Stokeslet flow field. In this situation, the Saffman force will direct to the channel centreline when particles lag the flow, or direct toward the channel walls when particles lead the flow.

\subsection{Wall-induced lift force}

In the previous section, we mainly talk about the first effect of walls. The existence of wall creates a velocity gradient (shear rate) of fluid, and possibly causes the rotation of spherical particles due to the shear. Thus, transverse Magnus force and Saffman force may act on the particles to migrate particles laterally. In addition, the second effect of walls is the change of the flow field around a particle in the presence of the walls. The net force on a particle near the wall differs from that of a particle in an unbounded flow. In order to eliminate effects of shear rate to the lateral lift force, one possible scheme is investigating the motion of particles near a wall in a stagnant fluid.

In general, the effect of a wall on the motion of immersed objects is to retard the motion of the object in both parallel and perpendicular directions, as well as exerting a transverse migration motion. Two distinct interactions between an immersed object and a wall exist: (i) The motion of the immersed object is primarily influenced by a single wall on one side of the object. Any other walls are too far from the immersed object to influence significantly its motion. In this case, the main effect of the wall are decelerating the particle and driving the immersed object away from the wall, Figure 1(c). It applies to the situation where the characteristic dimension of immersed particle is much less than the dimension of the flow channel. (ii) The immersed object is surrounded by the boundaries (walls) of the flow domain. Thus, the boundaries decelerate significantly the motion of the object. It happens if the characteristic dimension of the immersed particle is of the order of the dimension of flow channel, such as small bubbles moving in capillaries, and the surrounding walls will constrain the motion of immersed particles ${ }^{30}$.

First, we discuss the situation where the dimension of particles is much smaller than that of channel. The particles are primarily influenced by a single wall. The direction of the particle motion can be perpendicular or parallel to the walls. When the immersed sphere moves perpendicularly to a boundary, the effect of the boundary will retard the motion of the particle, which is equivalent to the increase of the drag coefficient. Brenner ${ }^{37}$ derived a first-order correction for the drag coefficient of a small sphere in the creeping flow moving towards a solid wall. 


$$
\begin{gathered}
f_{d}=\frac{16 \mu v_{t}}{a} f \\
f=\sinh \theta_{w} \sum_{n=1}^{\infty} \frac{n(n+1)}{(2 n-1)(2 n+3)} \times\left[\frac{2 \sinh \left[(2 n+1) \theta_{w}+(2 n+1) \sinh \left(2 \theta_{w}\right]\right.}{4 \sinh ^{2}\left[(n+0.5) \theta_{w}\right]-(2 n-1)^{2} \sinh ^{2}\left(\theta_{w}\right)}-1\right]
\end{gathered}
$$

where $\theta_{w}=\cosh ^{-1}\left(2 l_{w} / a\right)$ is a function of the ratio of distance from sphere centre to the wall to the particle diameter, $l_{w}$ is the distance of sphere centre to the channel wall, Figure 1(c). Equation (15) indicates that the hydrodynamic force on the particle increases significantly when the particle approaches the wall.

When the immersed sphere moves parallel to the boundary, a transverse lift force repels the particle away from the wall. Given the dimensionless distance $d^{*} \ll 1$, where $d^{*}=$ $\rho_{f} l_{w} v_{s} / \mu$, the lateral migration velocity can be expressed as ${ }^{38,39}$ :

$$
v_{z}=\frac{3}{64} R e_{s} v_{s}\left\{1-\frac{11}{32} d^{* 2}+\cdots\right\}
$$

where $v_{s}$ is the sedimentation velocity of the sphere in a quiescent fluid, and $R e_{s}$ is a Reynolds number defined by the sedimentation velocity and particle diameter, expressed as $R e_{s}=\rho_{f} a v_{s} / \mu$. Considering the implied condition of $d^{*} \ll 1$, it can be further simplified as:

$$
v_{z}=\frac{3}{64} R e_{s} v_{s}
$$

Equation (17) indicates that the migration velocity of a sphere is constant when it is in the vicinity of the wall. And this expression was experimentally validated by the data of Cherukat and McLaughlin ${ }^{40}$ up to $R e_{S}=3.0$.

In addition, when $d^{*} \gg 1$, the migration velocity then becomes ${ }^{39}$ :

$$
v_{z}=\frac{3}{16} R e_{s} v_{s}\left(d^{*-2}+2.21901 d^{*-5 / 2}+\cdots\right)
$$

It indicates that for large values of $d^{*}$ (large distance between sphere and wall or large sedimentation velocity of sphere), the migration velocity decreases continuously and tends to zero as $d^{*} \rightarrow \infty$.

In addition to the lateral lift force that repels particle away from the wall, the effect of the boundary will also retard the sedimentation motion of the particle, corresponding to an increased drag force. The drag force experienced by a sphere settling down in the neighbourhood of a plane wall $\left(\frac{a}{2 l_{w}} \ll 1 \ll \frac{1}{R e_{s}}\right)$ is ${ }^{39}$ :

$$
F_{d}=3 \pi \mu a v_{s}\left(1+\frac{3}{8} R e_{s}+\frac{9}{32} \frac{a}{l_{w}}+\cdots\right)
$$

At a relative large distance $\left(\frac{a}{2 l_{w}} \ll \frac{1}{R e_{s}} \ll 1\right)$, the drag force is expressed as ${ }^{39}$ :

$$
F_{d}=3 \pi \mu v_{s}\left\{1+\frac{3}{8} R e_{s}-0.095 \frac{a}{l_{w}}\left(\frac{1}{R e_{s}}\right)^{3 / 2}+\cdots\right\}
$$

In a bounded flow, when the characteristic dimension of the sphere is of the same order of magnitude as the size of the flow channel, the surrounding boundaries provide a physical constraint to the flow, and in general, the immersed objects move close to the centreline of 
the channel. Besides, the boundaries decelerate significantly the motion of the object. A wall drag multiplier of rigid sphere moving in a cylindrical tube was derived and expressed as ${ }^{30}$ :

$$
K_{\text {wall }}=\frac{F_{D}}{3 \pi a \mu v_{s}}=\left[1-2.0144 \delta+2.0888 \delta^{3}-6.948 \delta^{5}-1.372 \delta^{6}+\cdots\right]^{-1}
$$

In summary, the effects of walls on the motion of immersed spheres in a tube are to decelerate the motion of the sphere, and repel particles to the centreline of channel.

\subsection{Shear gradient lift force}

As discussed above, the particle will lag the flow due to the effects of wall. If the curvature of the undisturbed fluid velocity profile is zero, it becomes a simple shear flow, Figure 1(b). Then the pressure will be higher on the left, pushing particles to the centreline of channel. However, in the Poiseuille flow, the Stokeslet generated by the lag of the particle relative to the shear flow (slip-shear) may be balanced by an opposite Stokeslet originating from the curvature of the base flow. Furthermore, the curvature of fluid velocity profile can even reverse this trend. Figure 1(d) clearly shows that the magnitude of relative velocity of fluid to particle is much higher on the left side of particle than that on the right side, due to the parabolic nature of velocity profile. And it overwhelms the asymmetry caused by lag velocity. Similar to Saffman force, the dissymmetry of relative velocity causes a low pressure on the left/wall side, generating a shear gradient lift force that's opposite to the Saffman force. The Shear-gradient lift force leads particles migrating toward walls until the wall lift force repels and balances it ${ }^{32,33}$.

\subsection{Net inertial lift force}

For a neutrally buoyant rigid sphere flowing in a straight wall-bounded Poiseuille flow, besides a viscous drag force along the axis, there are four lateral forces acting on the sphere: Magnus force due to slip-rotation, Saffman force due to slip-shear, wall lift force due to the disturbance of flow field around particles from wall, and shear gradient lift force due to the curvature of undisturbed fluid velocity profile. Among them, Magnus force and Saffman force are often very small and negligible. Shear gradient lift force, directing particles toward channel walls, and wall lift force, repulsing particles toward channel centreline, are commonly recognised as the dominant effects for the lateral migration of the particle ${ }^{35}$. The balance of shear gradient lift force and wall lift force creates several equilibrium positions halfway between the channel walls and centreline, and this theory can explain the observation of Segre and Silberberg reasonably ${ }^{12,13}$, Figure 2(a). It should be noted that such a breakdown is rather unconventional, since an unbounded parabolic velocity profile cannot be realized in practice, but permits the separation of the effects due to the curvature of the undisturbed velocity profile and the wall-induced disturbance ${ }^{17}$.

Through the method of matched asymptotic expansions, Asmolov ${ }^{17}$ derived an analytical expression of the net inertial lift force acting on a small rigid sphere $(a / H \ll 1)$ in a Poiseuille flow:

$$
F_{L}=f_{L} \rho_{f} \gamma^{2} a^{4}
$$

The force can be further simplified as: 


$$
F_{L}=f_{L} \rho_{f} U^{2} a^{4} / H^{2}
$$

The hydraulic diameter $H$ is defined as $H=D$ for a circular channel ( $D$ is the diameter of circular cross-section) or $H=2 w h /(w+h)$ for a rectangular channel ( $w$ and $h$ correspond to width and height of the rectangular cross-section). The lift coefficient $f_{L}$ is a function of the particle lateral position $x$ and channel Reynolds number Re ${ }^{17,41}$, Figure 2(b). The lateral position $x_{e q}$ where $f_{L}=0$ corresponds to the inertial equilibrium position. It should be noted that the channel centreline $x=0$ is not a stable equilibrium position, since a little deflection from centreline will never return the particles back. It also has been found that $f_{L}$ decreases with increasing Re (or $U_{f}$ ), suggesting that inertial lift force scales less strongly than $U_{f}^{2}{ }^{4}$. Recently, Zhou and Papautsky ${ }^{42}$ derived a scaling for the lift coefficient based on their experimental results: $f_{L} \propto \frac{H^{2}}{a^{2} \sqrt{R e}}$. Although $f_{L}$ varies with Reynolds number, at $\operatorname{Re}<100$ that is typical for most microfluidic applications, the lift coefficient remains relatively constant, and can be approximated averagely as $f_{L}=0.5^{43}$.

For a finite-size particle $(0.05 \leq a / H \leq 0.2)$, particle would cause disturbance to the main channel flow. Di Carlo et al. ${ }^{44}$ calculated the inertial lift forces through finite element simulation, taking into account the finite-size effects of the suspended particles. The net force was scaled near the channel centre and channel wall respectively. Near the channel centre, where the effects of the wall are weak, the lift force scales as $F_{L} \propto \rho_{f} U_{f}^{2} a^{4} / H^{2}$. While near the channel wall, wall effects dominate, and it scales as $F_{L} \propto \rho_{f} U_{f}^{2} a^{6} / H^{4}$. 

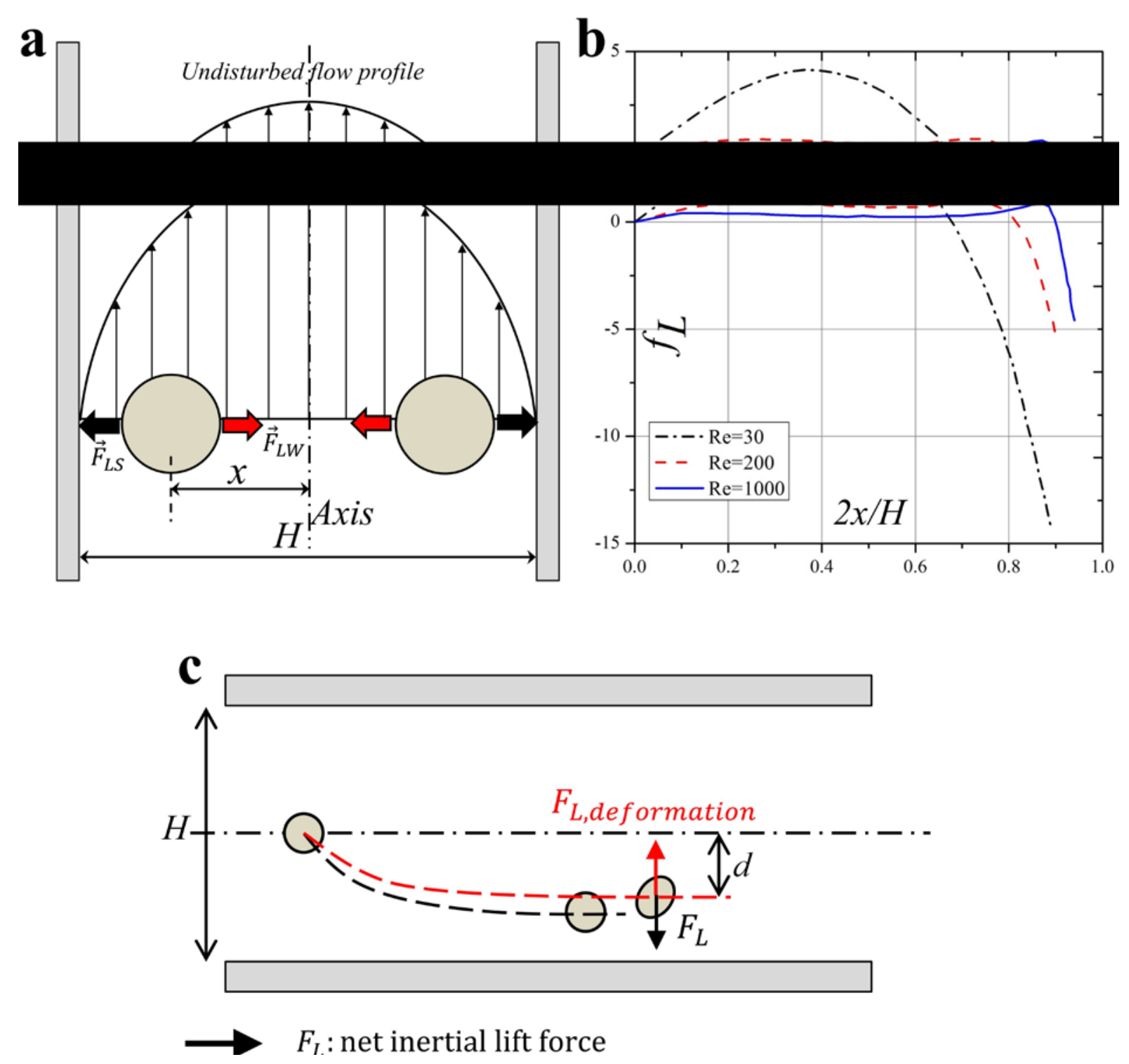

Figure 2 (a) Balance of shear gradient lift force and wall lift force results in the inertial equilibrium positions in a Poiseuille flow. (b) The net lift coefficient is a function of particle lateral position $x$ and Reynolds number Re ${ }^{22}$. (c) Deformability-induced lift force directs to the channel centreline, and shifts inertial equilibrium positions closer to the channel centre, compared with the rigid spherical particles ${ }^{45}$.

\subsection{Deformability-induced lift force}

Although solid rigid particles can be used as a simple model in the study of hydrodynamic behaviour of particles in a microchannel, the practical bio-particles such as cells and vesicles are not rigid but deformable. The deformability will induce additional lift forces on the particles. The deformability-induced lift force is perpendicular to the main streamline, and it is believed to be the effects of shape-change of particle and nonlinearities caused by the matching of velocities and stresses at the deformable particle interface ${ }^{35}$. Three dimensionless parameters can be used to characterize the relative deformation of a droplet: i) Weber number, $W e=\frac{\rho_{f} U^{2} a}{\sigma}$ (inertial stress vs. surface tension), where $\sigma$ is the surface 
tension; ii) Capillary number, $C a=\frac{\mu U a}{\sigma h}$ (viscous stress vs. surface tension); and iii) Viscosity ratio, $\lambda_{d}=\mu_{d} / \mu$, here $\mu_{d}$ is the dynamic viscosity of fluid inside the droplet ${ }^{35}$.

Chan and Leal derived an analytical expression for deformability-induced lift force, given the conditions that the drop or bubble is not too close to the walls ${ }^{46,47}$ :

$$
\begin{gathered}
F_{L, \text { deformation }}=\mu U a\left(\frac{a}{H}\right)^{2} \frac{d}{H} f\left(\lambda_{d}\right) \\
f\left(\lambda_{d}\right)=\frac{16 \pi}{\left(\lambda_{d}+1\right)^{3}}\left[\frac{11 \lambda_{d}+10}{140}\left(3 \lambda_{d}^{3}-\lambda_{d}+8\right)+\frac{3}{14} \frac{19 \lambda_{d}+16}{3 \lambda_{d}+2}\left(2 \lambda_{d}^{2}-\lambda_{d}-1\right)\right]
\end{gathered}
$$

where $d$ is the distance between drop and centre of channel. Furthermore, the condition of $\lambda_{d}<1$ or $\lambda_{d}>10$ was specified for the deformability-induced lift force directing towards the center of the channel ${ }^{47}$.

The experimental measurements of the inertial lift force by Di Carlo et al. ${ }^{44}$ was used to extract an equation for inertial lift force near channel centre where $d / H<0.1$. The negative sign indicates that this force, $F_{L \text {,inertial }}^{\text {center }}$, is directed towards the walls.

$$
F_{L, \text { inertial }}^{\text {center }}=-10 R_{p} \mu U a\left(\frac{d}{H}\right)
$$

When both drop deformation and inertial lift forces are small (for $\mathrm{Ca}<0.01$ and $R_{p}<$ 0.01 ), Stan et al. ${ }^{46}$ introduced an empirical formula for net lift force on drops or bubbles as:

$$
F_{L, \text { empirical }}=C_{L} \mu U a\left(\frac{a}{H}\right)^{3}\left(\frac{d}{H}\right)
$$

Here lift coefficient $C_{L}$ must be determined from experimental measurements for each pair of the continuous and dispersed phases. And $C_{L} \sim 31.25$ can be used for the order-of-magnitude estimate ${ }^{46}$.

Deformability-induced lift force can be used to separate and enrich malaria-infected red blood cells (iRBCs) from normal healthy RBCs ( $h$ RBCs) for the diagnosis of malaria. The parasite releases proteins that trigger the cross-linking of the spectrin network in the $i$ RBC's phospholipid bilayer membrane, thus increases the rigidity of the $i$ RBCs. Normal $h$ RBCs are more deformable than $i$ RBCs, migrating towards to the centre of the channel. Due to the massive hydrodynamic interactions and mechanical collisions between the RBCs in high haematocrit (Hct) blood, stiffer malaria iRBCs are displaced towards the sidewalls, and can be depleted and enriched by bifurcated outlets ${ }^{48}$.

In inertial microfluidics, Hur et al. ${ }^{45}$ found that centre-directed deformability-induced lift force shifts inertial equilibrium positions a little closer to the channel centreline than that of rigid particles, Figure 2(c). By the combination of size and deformability, circulating tumor cells (CTCs) with more deformability than the cells from the same organ, has been demonstrated to be separated and enriched from peripheral blood ${ }^{45}$.

Besides particle deformability, the shape of particles ${ }^{49}$ and the properties of medium ${ }^{50}$ also impact the inertial migration and equilibrium positions, which will not be discussed here. A summary of particle kinetics in inertial microfluidics is shown in Figure 3. 


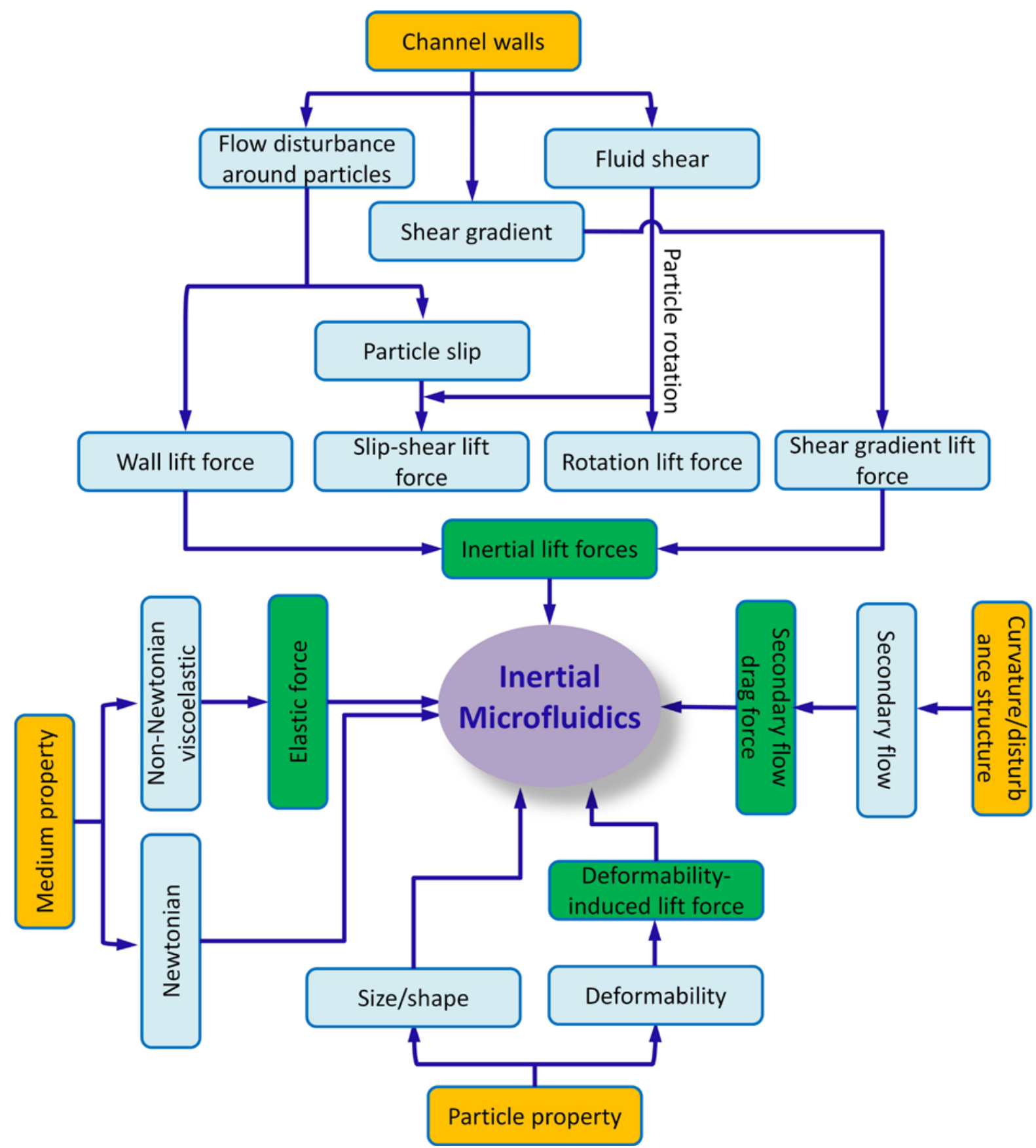

Figure 3 Outline of particle kinetics in inertial microfluidics. There are four aspects impacting inertial focusing in inertial microfluidic systems: channel walls, channel curvature or disturbance structure, particle and medium property. Channel walls create wall lift force, shear gradient lift force, slip-shear lift force and rotation lift force on particles, while front two lift forces are widely recognised as responsible for inertial migration phenomenon. Channel curvature or disturbance structure generates secondary flow, which assists inertial focusing process and modifies final inertial focusing positions. Besides, the properties of particle (e.g. size, shape and deformability) and medium (Newtonian, Viscoelastic) also influence the final inertial focusing positions.

\section{THE RECENT PROGRESS OF INERTIAL MICROFLUIDICS}

According to the structure of the functional microchannel, the reported inertial microfluidic devices can be categorised according to their channel structure as: (i) straight channels, (ii) 
spiral channels, (iii) straight channels with pillar arrays or expansion-contraction arrays, and (iv) serpentine channels. In this chapter, we review the current progress of inertial microfluidics, including the phenomenon and possible explanation of inertial migration in different structured micro-channels, as well as their application in biomedicine and disease diagnosis. The characteristics of inertial forces in different structured channels were summarized in Supplementary Table 1.

\subsection{Straight channel}

In a straight channel with a circular cross-section, the randomly distributed particles migrate laterally to a narrow annulus at about 0.6 times of the channel radii form the axis ${ }^{12,22}$, as depicted in Figure 4(a). However, in a straight channel with a rectangular cross-section, which is the most widely used structure due to the limitation of microfabrication, the situation becomes more complex. In a square straight channel ( $A R=$ height/width=1), particles normally focus to four equilibrium positions ${ }^{51}$, facing the centre of each wall, Figure 4(b). Additionally, a further reduction to two equilibrium positions happens in a low aspect ratio $(A R \approx 0.5)$ channel, particles focus at the centre about $0.2 H$ away from the long walls (see Figure 4(c) ${ }^{42,52,53}$. One explanation about this equilibrium positions' reduction was given by Zhou and Papautsky ${ }^{42}$ by a two-stage migration model which is illustrated in Figure 4(c).

In a straight channel, particles' lateral migration velocity $\left(U_{L}\right)$ and the minimum channel length $\left(L_{\text {min }}\right)$, which is the minimum length that is required for particles to migrate to their inertial equilibrium positions, can be derived by balancing the net inertial lift force with Stokes drag (see Figure 4(d)) ${ }^{41}$ :

$$
\begin{gathered}
F_{\text {stks }}=3 \pi \mu a U_{L} \\
U_{L}=\frac{F_{L}}{3 \pi \mu a}=\frac{\rho_{f} U^{2} a^{3}}{6 \pi \mu H^{2}} \\
L_{\min } \approx \frac{H}{2 U_{L}} * U=\frac{3 \pi \mu_{f} H^{3}}{\rho_{f} U a^{3}}
\end{gathered}
$$

Two dimensionless Reynolds numbers can characterize the lateral migration of particles in straight channel: channel Reynolds number Re describing the ratio between inertial force and viscous force of fluid in a flow, and particle Reynolds number $\mathrm{R}_{\mathrm{P}}$ additionally considering the size ratio of particle to channel.

$$
R_{P}=\operatorname{Re} \frac{a^{2}}{H^{2}}=\frac{\rho_{f} U a^{2}}{\mu H}
$$

When $\mathrm{R}_{\mathrm{P}}<<1$, particles are subjected to the dominant viscous drag to follow fluid streamlines. However, increasing $\mathrm{R}_{\mathrm{P}}$ to the order of 1 , inertial lift forces become dominant and lateral migration of particles across the fluid streamlines becomes obvious ${ }^{43}$. 

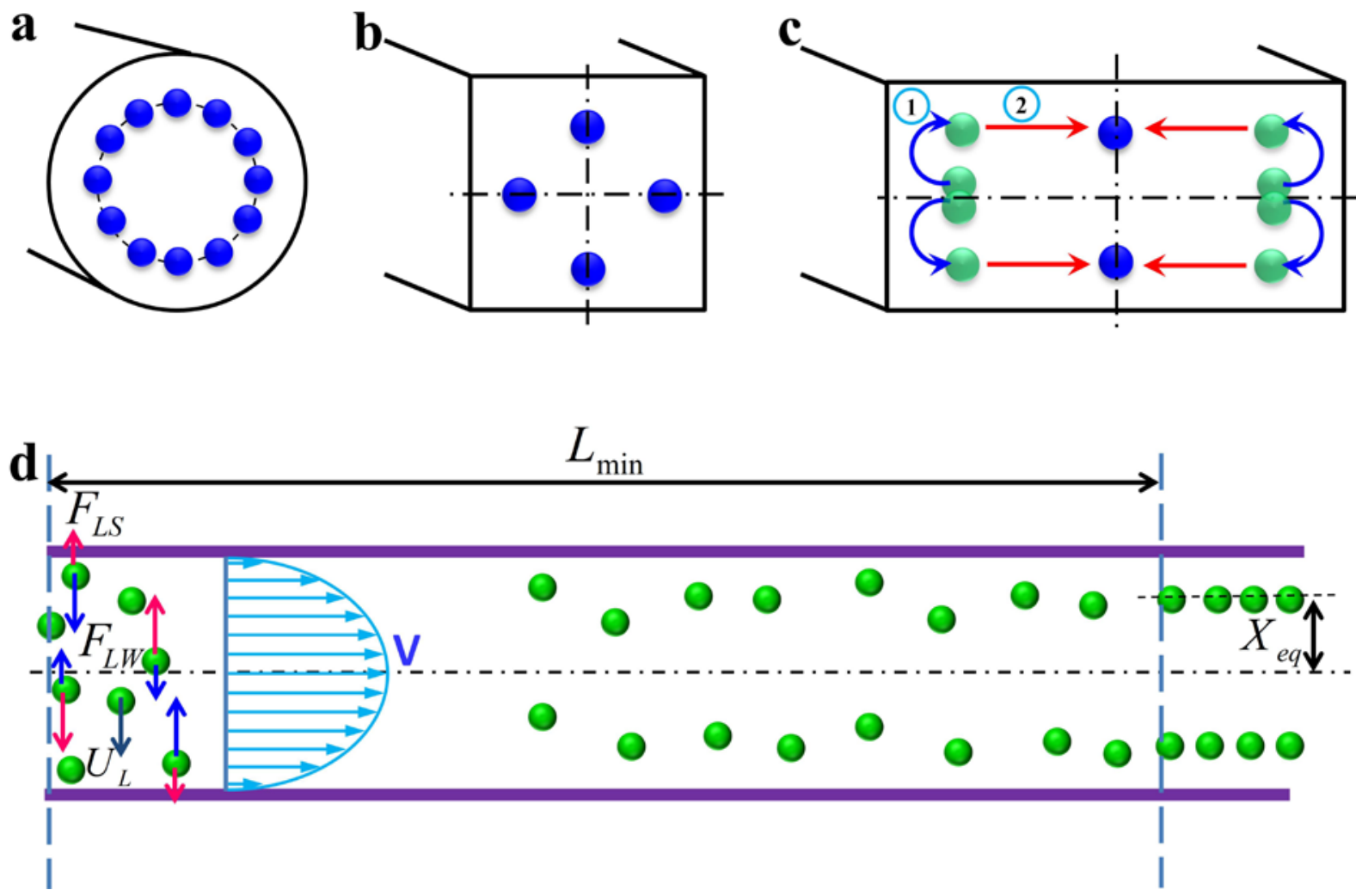

Figure 4 Inertial equilibrium positions in a straight channel with different cross sections: (a) Circular cross section; (b) square cross-section, and (c) rectangular cross section with low aspect ratio $(\approx 0.5)$. Illustration of two-stage migration model proposed by Zhou and Papautsky ${ }^{42}$. (d) The lateral migration speed $U_{L}$ and minimum channel length for particle focusing $L_{\min }$.

Although straight channel is often employed as a basic model to investigate the fundamental mechanism of inertial migration due to its simplicity ${ }^{18,42,44,54-56}$, this channel type has been utilized for a variety of applications. Hur et al. ${ }^{57}$ demonstrated the purification of adrenal cortical progenitor from digestions of murine adrenal glands utilizing hydrodynamic inertial lift forces in a straight micro-channel with high aspect ratio, Figure 5(a). The inertial focusing position depends on the cell size, where larger cells are closer to the channel center, and smaller cells are closer to the channel walls. Differentially focused cells can be collected at designated outlets based on apparent diameter. 

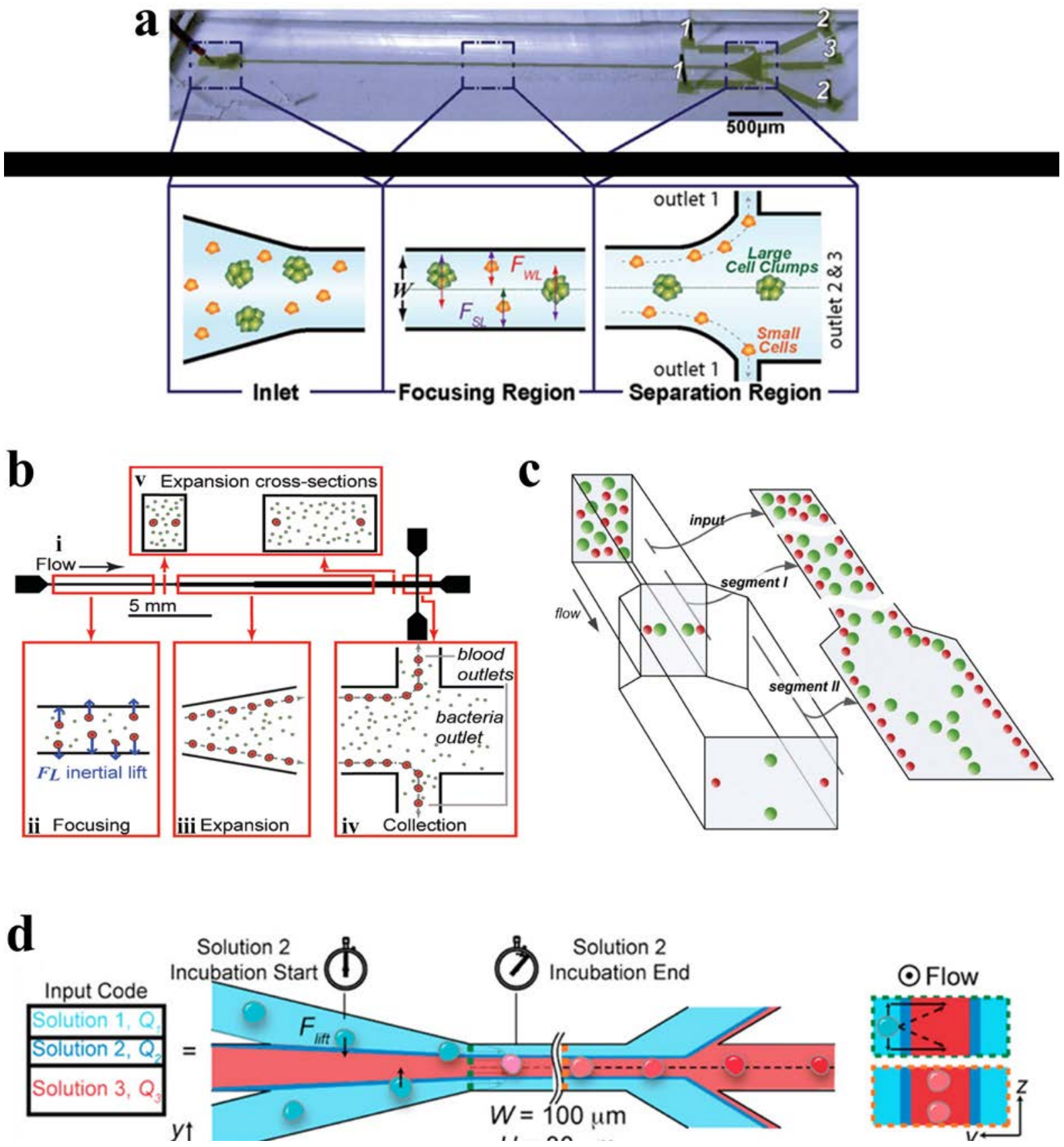

Figure 5 (a) Label free isolation of adrenal cortical progenitor cells by size-based differential inertial focusing in a straight channel. Reproduced from Ref. ${ }^{57}$, an open-access article. (b) Separation of pathogenic bacteria from diluted blood in a series-connected straight channel which utilizes a unique differential transit time by sizedependent inertial lift forces. Reproduced from Ref. ${ }^{58}$ with permission from John Wiley and Sons. (c) Complete separation of particles in a cascaded channel with two straight segments with different aspect ratios (ARs). Reproduced from Ref. ${ }^{59}$ with permission from the Royal Society of Chemistry. (d) Rapid inertial solution exchange in a straight channel for controlled interaction of reagents with cells and particles. Reproduced from Ref. ${ }^{60}$ with permission from American Chemical Society Copyright (2014).

Mach and Di Carlo ${ }^{58}$ reported a massively parallelized microfluidic device that passively separates pathogenic bacteria from the diluted blood (Figure 5(b)). The device consists of 40 single straight micro-channels placed as a radial array. Each channel consists of three segments with different cross-sections, which uses a unique differential transit time by sizedependent inertial lift forces to obtain cell separation. The authors demonstrated that more 
than $80 \%$ pathogenic bacteria can be removed after passing two times of the same system. The parallel device can process $240 \mathrm{~mL} / \mathrm{h}$ with an extreme high throughput of 400 million cells per minute. Later, Zhou and colleagues ${ }^{59}$ utilized a modified design on size and length for their cascaded straight channels, as shown in Figure 5(c). The separation concept is based on the theory of the two-stage inertial migration, which permits precise prediction of particle or cell position within the micro-channel. Randomly-distributed particles first flow through a high $A R$ channel (segment I) where particles are focused at the half of channel height near two sidewalls. Then the channel expands into a low $A R$ channel (segment III), which modifies equilibrium positions to the centres of top and bottom walls. Since larger particles have a much higher migration velocity, they reach the relocated points very quickly. In contrast, lift forces on smaller particles are too weak to alter their lateral position. This effect enables a separation of particles by size. After this modification, a much higher separation efficiency ( 99\%) and purity ( 90\%) were achieved in their work.

Manual concentration, staining and washing procedures for cellular sample are routinely conducted by cytopathologists as a means of diagnosing malignancies and other diseases. However, current approaches for chemical treatments of cells and chemical reactions typically operate on slow time scales ( seconds to minutes), limiting the realm of fast molecular events. Inertial lateral migration has been subtly employed as a helpful tool to mediate millisecond reaction time around particles and cells ${ }^{60}$, as shown in Figure 5(d). The main transfer channel is designed with a low aspect ratio, so particles will migrate to the lateral centre of a channel. The channel has three inlets. Particles suspension is infused from the two side inlets, while the reagent is pumped in from the central inlet. Lateral migration of particles from original medium to the middle stream initiates the contact of particles/cells with chemical reagent, and duration of particles within middle stream mediates the reaction time. The proposed microfluidic system can perform several functions in the sample preparation for cytopathology that (i) automates colorimetric staining on-chip, (ii) images cells in flow, and (iii) provides additional quantitative analyses of captured images to aid cytopathologists ${ }^{61}$. In addition, ordering particles along a specific stream within a simple straight microchannel could also be integrated with optical detector to form an on-chip flow cytometer system. In another study, Hur et al. ${ }^{62}$ proposed an extremely high-throughput on chip imaging flow cytometry, consisting of 256 high-aspect parallel straight channels and high-speed optical camera, promising interrogation rates up to 1 million cells per second.

In general, a straight channel has the advantage of simplicity and ease of operation. Therefore, the mechanism of inertial migration phenomenon in a straight channel is relatively clear and has already been studied extensively. However, since $F_{L} \propto H^{-2}$, the sizes of channel crosssection are normally restricted to provide enough lateral lift forces. In addition, the channel is relatively long, which would add up the flow resistance and lead to a large device footprint. In the following section, we will see that, the introduction of a secondary flow by channel curvature or obstacle structure will not only aid the inertial migration progress, but also modify the final equilibrium positions. However, its mechanism becomes more complex and demands more rigorous explanation. 


\subsection{Spiral channel}

Introduction of channel curvature along a single constant direction forms a spiral channel. When fluid flows through a curved channel, a secondary flow arises due to the velocity mismatch in the downstream direction between fluid in the central and near-wall regions. The fluid elements near the channel centreline have larger inertia and would tend to flow outward around a curve due to the centrifugal effect, creating a pressure gradient in the radial direction within the channel. In a fully-bounded channel, due to the centrifugal pressure gradient, relatively stagnant fluid near the walls re-circulates inward, finally forming two symmetric circulating vortices ${ }^{4}$, Figure 6 (a). The parameters that influence the distribution and strength of the secondary flow in a curved channel include Dean number $K$, Reynolds number Re and aspect ratio of the channel $A R$. Dean number $K$ is a function of Reynolds number Re, the hydraulic diameter for the rectangular channel $H$ and the radius of curvature $R^{23}$ :

$$
K=(H / 2 R)^{1 / 2} R e
$$

Following Squires and Quake ${ }^{63}$, the secondary flow velocity scales as

$$
U_{D} \sim K^{2} \mu /(\rho H)
$$

Furthermore, the magnitude of secondary flow $U_{D}$ can be approximated as ${ }^{64,65}$ :

$$
U_{D}=1.8 \times 10^{-4} \times K^{1.63}
$$

In addition, the ratio of channel dimension to radius of curvature $\delta=H / 2 R$, Dean number $K$ as well as the aspect ratio $A R$ have important effects on secondary flow distribution ${ }^{23}$.

Particles flowing in a curved channel with finite inertia will experience both inertial lift forces and secondary flow drag. In most cases, the density of particle is very close to that of fluid, so the effect of particle centrifugal force around channel curvatures is negligible. When the channel curvature is along a single direction, the curved channel is a spiral channel. And the direction of secondary flow within each cross-section is constant, although there may be a little variation on its magnitude due to the change of channel curvature. By a first order approximation, one can make an assumption that the effects of inertial migration and secondary flow act in superposition on particles in spiral channels, Figure 6(b). A particle held stationary at inertial equilibrium positions experiences a secondary flow drag whose magnitude is directly proportional to the local secondary flow velocity ${ }^{4}$. This secondary flow drag force acts to entrain particles within the streamline of symmetrically rotating vortices, which is primarily an effect of mixing. In contrast, inertial lift forces tend to hold particles at specific equilibrium positions within channel cross-section. Therefore, the order of magnitude scaling between inertial lift forces and Dean drag $R_{f}=a^{3} R / H^{3}$ determines the final behaviour of the suspended particles. This dimensionless parameter is useful for the prediction of particle behaviour in curved channels.

At limiting conditions where (i) $R_{f} \rightarrow 0$, Dean drag force dominates the behaviour of particles, particle streams neglect inertial equilibrium positions, and remain entrained within the secondary flow streamlines; and (ii) $R_{f} \rightarrow \infty$, inertial lift force is dominant, particles migrate to the inertial equilibrium positions independent of the secondary flow ${ }^{4}$. For most 
cases, in the intermediate range of $R_{f}$, inertial equilibrium positions will be modified by the secondary flow, Figure 6(b), leading to very intriguing new focusing phenomenon. It has been noted that $R_{f}$ is dependent on particle size, so that two different-sized particles may experience different forces in the same curved channel. Therefore, it would be possible to separate particles by size in spiral channels.

Yoon et al. ${ }^{66}$ proposed another kind of particle separation in a curved channel. In their work, the separation was achieved by the net effect of secondary flow velocity distribution, rather than the theory of the balance between secondary flow drag and inertial lift forces. Larger particles with diameter larger than $0.72 h$ experience a net secondary flow drag directing outward to the channel curvature, while smaller particles with diameter less than $0.27 \mathrm{~h}$ experience a net secondary flow drag inward, Figure 6(c) and (d). Therefore, particles can be separated based on altered net direction of secondary flow drag on different-sized particles.
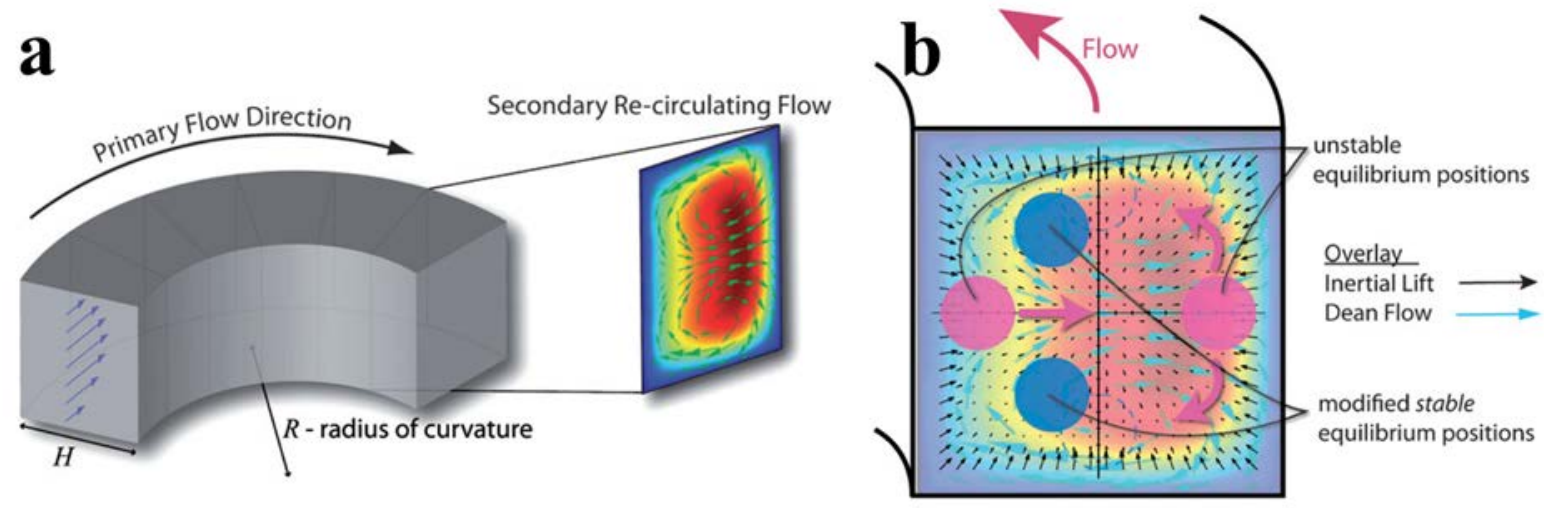

c

d
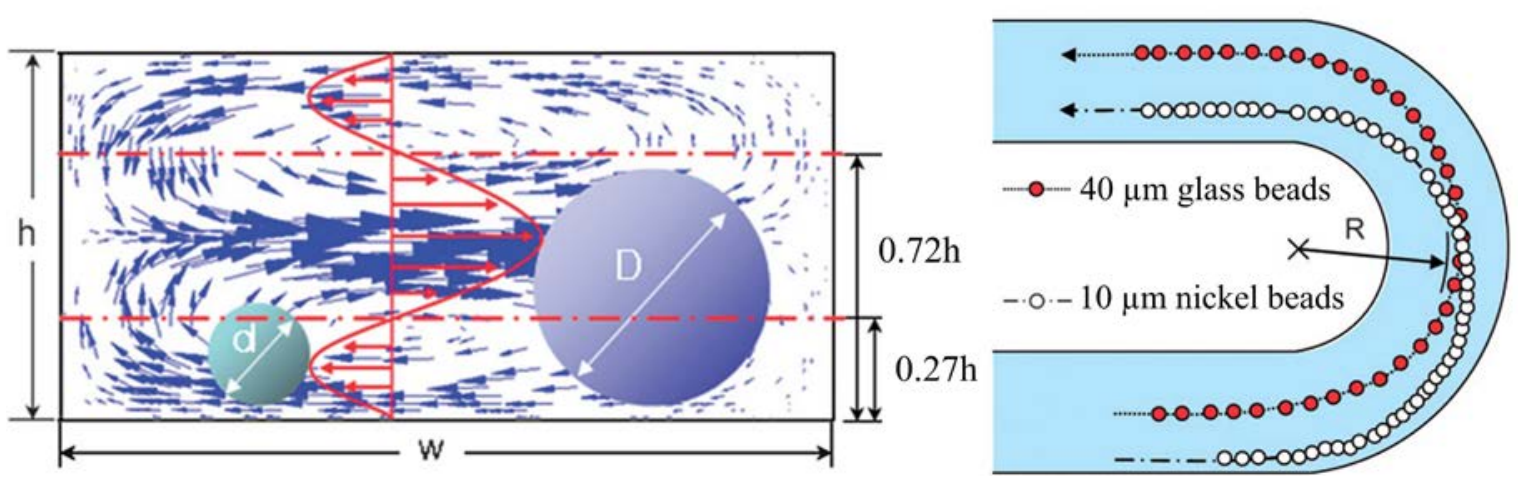

Figure 6 (a) Dean flow with two counter-rotating vortices are created in curved channels. Reproduced from Ref.

${ }^{4}$ with permission from the Royal Society of Chemistry. (b) Superposition of inertial lift force and Dean flow in a curved channel modifies the number and position of the inertial equilibrium positions. Reproduced from Ref. ${ }^{4}$ with permission from the Royal Society of Chemistry. (c) - (d) Size-based separation by the different directions of secondary flow drag force exerted by the velocity distribution. Reproduced from Ref. ${ }^{66}$ with permission from the Royal Society of Chemistry.

Spiral channels were investigated extensively for particle ordering and separation by Papautsky’s group ${ }^{67,68}$, Go’s group ${ }^{66}$, Jiang's group ${ }^{69,70}$ and Han’s group ${ }^{71,72}$, etc. Bhagat et al. ${ }^{64}$ proposed a sheath-less, on-chip flow cytometry system based on the principle of 
Dean coupled inertial microfluidics, Figure 7(a). The microfluidic flow cytometry system could provide a throughput of 2,100 particles per second. Furthermore, Kemna et al. ${ }^{65}$ reported an integrated microfluidic system that combines cell-ordering in spiral channel with droplet microfluidic generator. Cells were focused in three dimensional along a single particle chain and ordered with a precise longitudinal space, Figure 7(b). Single cell encapsulation was successfully demonstrated downstream with efficiency about $80 \%$. Such a system is very promising in the applications such as cell-based assays, drug screening and cell printing technologies.

Passive separation and sorting of particles/cells is one of superior advantages for spiral microchannels, benefiting from the distinct size dependence characteristics of secondary flow and inertial lift forces. In another work, Bhagat and colleagues ${ }^{67}$ demonstrated a complete separation of $7.32 \mu \mathrm{m}$ and $1.9 \mu \mathrm{m}$ polystyrene particles at Dean number of 0.47 in a 5-loop spiral microchannel. Later, Kuntaegowdanahalli and co-workers ${ }^{68}$ from the same group extended the separation capability of spiral channels. The authors demonstrated continuous separation of triplet polystyrene beads (10, 15 and $20 \mu \mathrm{m}$ in diameter) with an efficiency of $90 \%$ and a throughput of $1 \times 10^{6}$ cells/min in a spiral channel, Figure 7 (c). Furthermore, this separation ability was employed to separate different-staged cells for cell cycle synchronization, which is essential for studying cellular properties, biological processes and elucidating genetic regulatory mechanisms and events involved in each phase prior to cell division ${ }^{73}$.

Cancer, also known as a malignant tumour, is a group of diseases involving abnormal cell growth with the potential to invade or proliferate in other organs of the body. Metastases from primary tumours are the leading causes of death ( 90\%) for nonhematological cancers ${ }^{74}$. During the progression of metastasis, cancer cells escaped from solid tumours and enter the bloodstream, becoming circulating tumour cells (CTCs), which hold a great potential to serve as important biomarkers for early diagnosis of cancer metastases, as well as cancer prognosis and therapy monitoring. CTCs analyses are considered as a real-time "liquid biopsy”, which is much less invasive than the current method for cancer diagnosis requiring invasive biopsy followed by molecular analysis. However, CTCs are extremely rare, comprising only a few out of one billion haematological cells in blood, making their isolation and characterization an extreme technological challenge.

In an intriguing work, Hou et al. ${ }^{75}$ employed a spiral channel to isolate CTCs from blood under the assistance of a sheath flow, and it achieved a recovery rate of more than $85 \%$. This CTCs isolation platform is working in a label-free and clog-free continuous manner, significantly reducing the capturing costs and alternation of CTCs' morphology. In addition, a clinical validation with positive detection of CTCs from all the patients' blood samples was reported. Meanwhile, Sun and colleagues ${ }^{69,70}$ reported a passive double spiral microfluidic device, Figure 7(d), which can separate and enrich tumour cells (MCF-7 and Hela cells) from diluted whole blood with a throughput of $3.33 \times 10^{7}$ cell $/ \mathrm{min}$. Compared to the single spiral microchannel, the double spiral device was claimed to provide better focusing behaviour of small blood cells and improved separation efficiency. To further scale up the throughput of spiral channels, parallelization technology must be employed. Warkiani et al. ${ }^{76}$ reported a 
multiplexed microfluidic device stacked by three spiral channels along vertical direction for ultra-high throughput CTCs capturing and enrichment, Figure 7(e). The advantages of very fast processing speed $(7.5 \mathrm{ml}$ blood in less than $10 \mathrm{~min})$ and the ability to collect more CTCs from larger blood volumes make it very promising for a range of potential genomic and transcriptomic applications.

Guan and co-workers ${ }^{77}$ introduced a novel spiral micro-channel with a trapezoidal crosssection and it showed a higher separation resolution than those with rectangular cross-section. Their study found that particle focusing in spiral channel with trapezoidal cross-section is sensitive to particle size and flow rate, and exhibits a sharp transition at a size-dependent critical flow rate. Wu et al. ${ }^{72}$ applied this novel spiral channel for the separation of Leukocytes from blood sample. At the outlet, the larger white blood cells focus near the inner walls, while smaller red blood cells are trapped at the core of Dean vortex near the outer wall. An enhanced separation resolution and efficiency (>80\%) was demonstrated. Later, isolating CTCs from blood samples of cancer patients was demonstrated using the similar slanted spiral micro-channels ${ }^{71}$ by the same group, Figure $7(\mathrm{f})$. The device has successfully isolated and recovered more than $80 \%$ of the tested cancer cell lines spiked in $7.5 \mathrm{ml}$ blood within 8 min with high purity (400-680 WBCs $\mathrm{ml}^{-1}$; 4log depletion of WBCs). In the initial clinical investigation, the trapezoid chip successfully isolated CTCs from 10 out of 10 (100\%) patients with advanced stage metastatic breast and lung cancer (3-125 CTCs/ml), and it allowed extensive heterogeneity studies via immunostaining and DNA FISH analysis. 

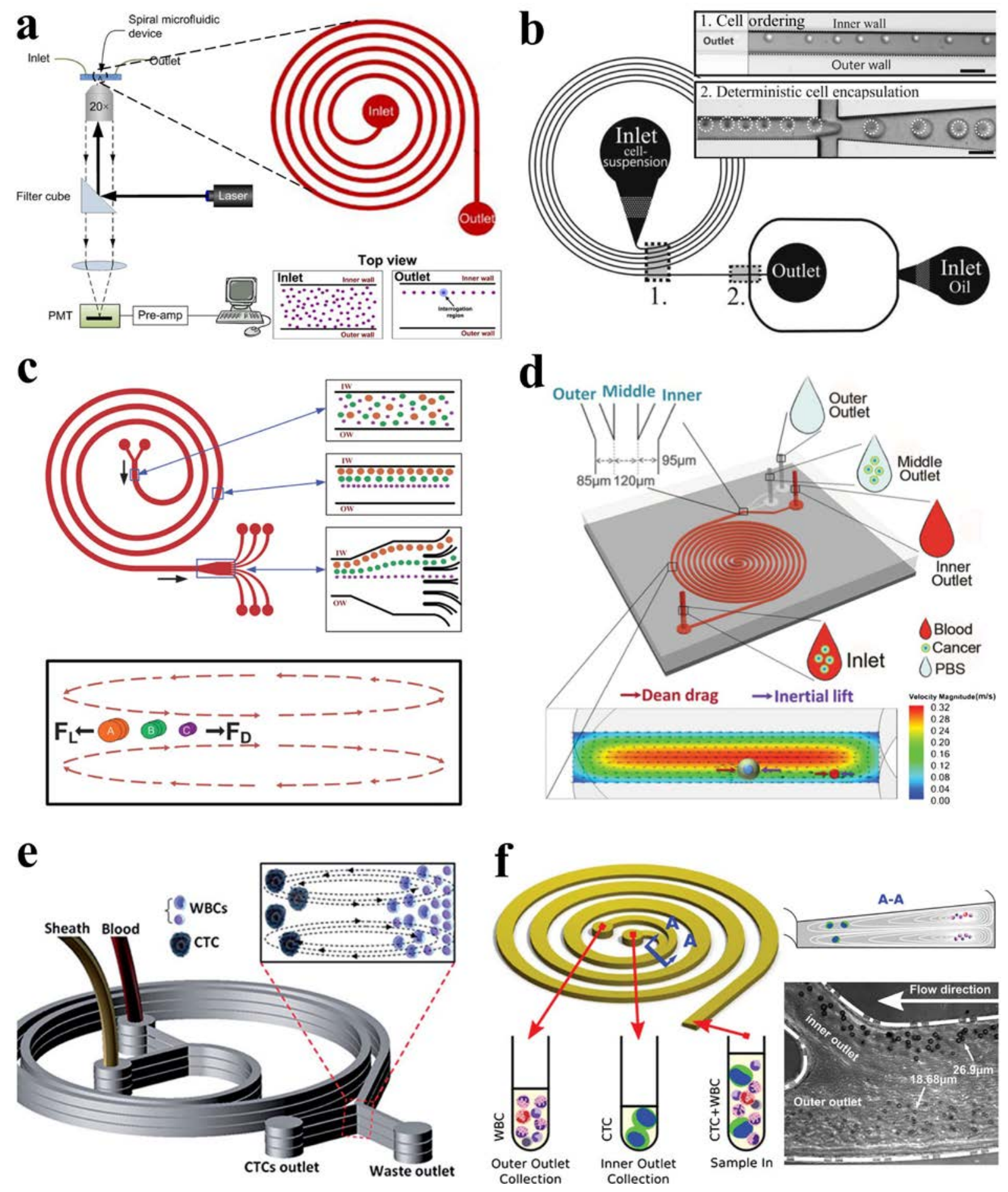

Figure 7 (a) Schematic of a microfluidic flow cytometry, in which spiral channel is applied as a particle focuser to provide high-throughput sheath-less 3-D focusing, followed by the downstream laser induced fluorescence setup for particle detection and counting. Reproduced from Ref. ${ }^{64}$ with kind permission from Springer Science \& Business Media. (b) Single cell encapsulation by droplets in a spiral channel. The spiral channel promotes single cell stream ordering with a precise longitudinal spacing, and the downstream droplet generator encapsulates cells into the droplets. Reproduced from Ref. ${ }^{65}$ with permission from the Royal Society of Chemistry. (c) Continuous triplet-particle separation in a spiral channel. Reproduced from Ref. ${ }^{68}$ with permission from the Royal Society of Chemistry. (d) Double spiral microchannel for tumour cell separation and enrichment. Reproduced from Ref. ${ }^{69}$ with permission from the Royal Society of Chemistry. (e) A multiplexed microfluidic device stacked by three spiral channels for ultra-high throughput CTCs capturing and enrichment ${ }^{76 \text {, }}$ 
78. (f) Ultra-fast, label-free enrichment of CTCs from blood samples by a spiral microfluidic device with trapezoid cross-section. CTCs are focused near the inner wall due to the combination of inertial lift force and Dean drag force, while white blood cells and platelets are trapped inside the core of the Dean vortex closer to the outer wall ${ }^{71}$.

\subsection{Straight channel with pillar arrays or expansion-contraction arrays}

Besides curvature, introduction of disturbance obstacles into straight channels will also induce convective secondary flow, which was first reported to enhance mixing effects by continuously splitting and redirecting fluid streams ${ }^{24}$. By patterning a contraction-expansion array on single side of a straight channel, Park's group ${ }^{79}$ successfully demonstrated three dimensional single stream particle focusing under a sheath flow. At the entrance of the contraction region, the centrifugal forces induce counter-rotating secondary flow, enveloping a sample flow with a sheath flow in three dimensions. Besides particle focusing, a series of particle separation in this kind of contraction-expansion array (CEA) channels were also reported by the same group, including separating polystyrene beads of $4 \mu \mathrm{m}$ and $10 \mu \mathrm{m}$ in diameter ${ }^{53}$, blood plasma from red blood cells ${ }^{80}$ and cancer cells from whole blood ${ }^{81}$, Figure 8(a). Expansion-contraction array induces Dean-like secondary rotating flows, working together with inertial lift forces on the suspended particles. Large particles or cells such as cancer cells are influenced dominantly by the inertial lift forces, migrating towards contraction-expansion side, while small particles or cells (e.g. red blood cells and white blood cells) are dominated by Dean drag, shifting towards opposite side. Finally, it enables a sizebased particle/cell separation and enrichment ${ }^{81}$.

The contraction-expansion array can also be patterned on two sides of the channel. Park et al. ${ }^{82}$ investigated particle inertial focusing in a straight channel patterned with symmetrical expansion-contraction arrays on both sides (multi-orifice microchannel), Figure 8(b). This device is working without any sheath flow. The ordered particle distribution can be achieved at central or side regions according to a particle Reynolds number $R_{\mathrm{p}}$. Generally, particles are focused at two side positions with $R_{\mathrm{p}}$ range of $\sim 0.8$ to 2.3 and at the centreline with the $R_{\mathrm{p}}$ range of 3.0 to 3.5. The mechanism of these focusing patterns is relying on the combination of inertial lift force and momentum-change-induced inertial force generated in the contraction-expansion region. The trajectory mismatch between particles and fluid element around the contraction-expansion region induces the lateral drift of the equilibrium position. The extent of this lateral drift is variable according to particle size and flow rate. In the Reynolds number range of 63 to 91, large polymer particles $(\sim 15 \mu \mathrm{m})$ were aligned at the centreline of the outlet, whereas small particles $(\sim 7 \mu \mathrm{m})$ remained along both sidewalls. Therefore, size-based separation of particles in this kind of multi-orifice channel is achieved, and termed as Multiorifice Flow Fractionation (MOFF) ${ }^{83}$. The MOFF has several advantages such as continuous, label-free, sheath-less, and non-intrusive with minimal power consumption. However, it is limited by the relatively low recovery yield. Although the recovery yield may be increased by adjusting parameters such as the Reynolds number to enhance central focusing, poor purity inevitably followed. Both high recovery yield and high purity cannot be warranted at the same time. Therefore, Sim et al. ${ }^{84}$ presented a multi-stage multi-orifice flow fractionation (MS-MOFF), which is made by combining three multi-orifice 
segments, 3 inlets, 3 filters and 5 outlets. It could improve recovery and minimize loss of purity by collecting and re-separating non-selected particles from the previous separation. The final recovery rate was successfully increased from $73.2 \%$ to $88.7 \%$ without significant compromise on purity. By combining multi-orifice flow fractionation (MOFF) with dielectrophoresis (DEP) techniques, Moon et al. ${ }^{85}$ successfully separated human breast cancer cells (MCF-7) from spiked blood sample, Figure 8(c). The inertial separation by MOFF takes advantage of the high-throughput filtration of blood cells, and the serially connected DEP separator works as a precise post-processor to further enhance the separation efficiency and purity.

Diagnosis of malaria at the early stage of infection is challenging due to the difficulty in detecting the low abundance of parasites from blood. Polymerase chain reaction (PCR) method can be especially useful to detect low parasitemia levels, but the efficiency is discounted by many factors, such as limited specificity of primers, presence of PCR inhibitors in blood serum and DNA contamination from nucleated blood cells. Recently, Warkiani and co-workers ${ }^{86}$ presented an improved detection method for malaria by the combination of inertial microfluidic processing and PCR detection, Figure 8(d). In the symmetrical contraction-expansion channel, particles larger than $4 \mu \mathrm{m}$ such as WBCs were focused along the channel walls and isolated via the peripheral outlets. The malaria parasites, on the other hand, were unfocused and distributed in all the three outlets. Therefore, enriched and purified blood sample would promote more reliable and specific PCR-based detection. 
a

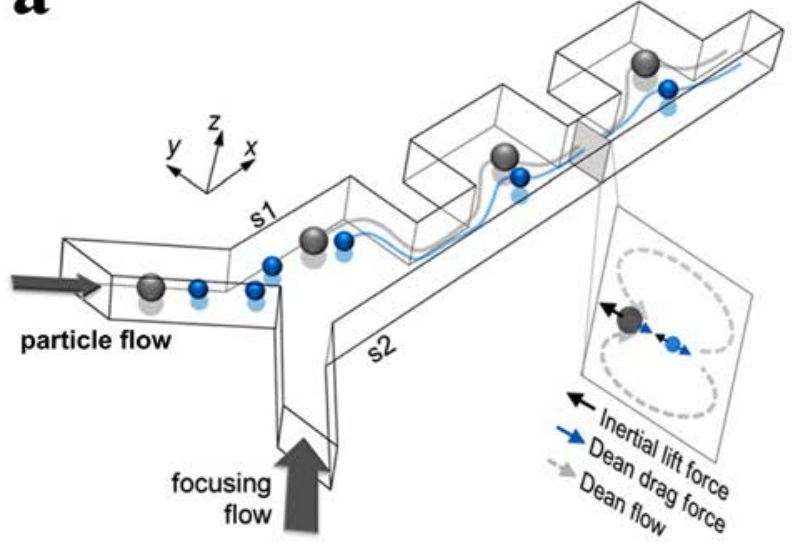

b

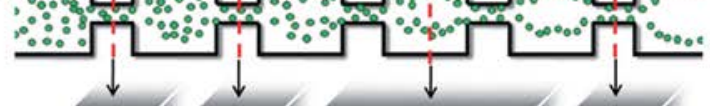

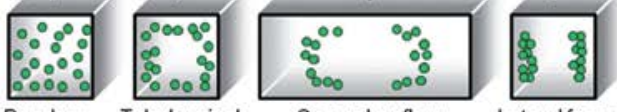

Random Tubularpinch Secondary flow Lateral focus

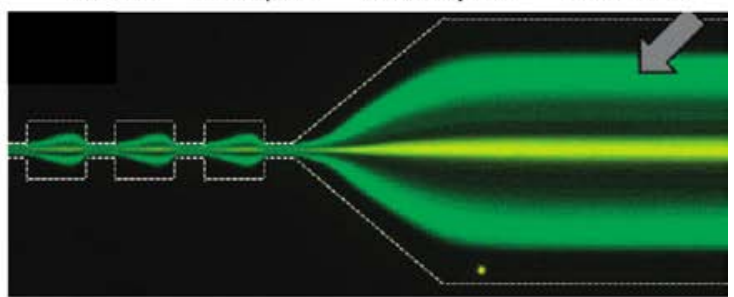

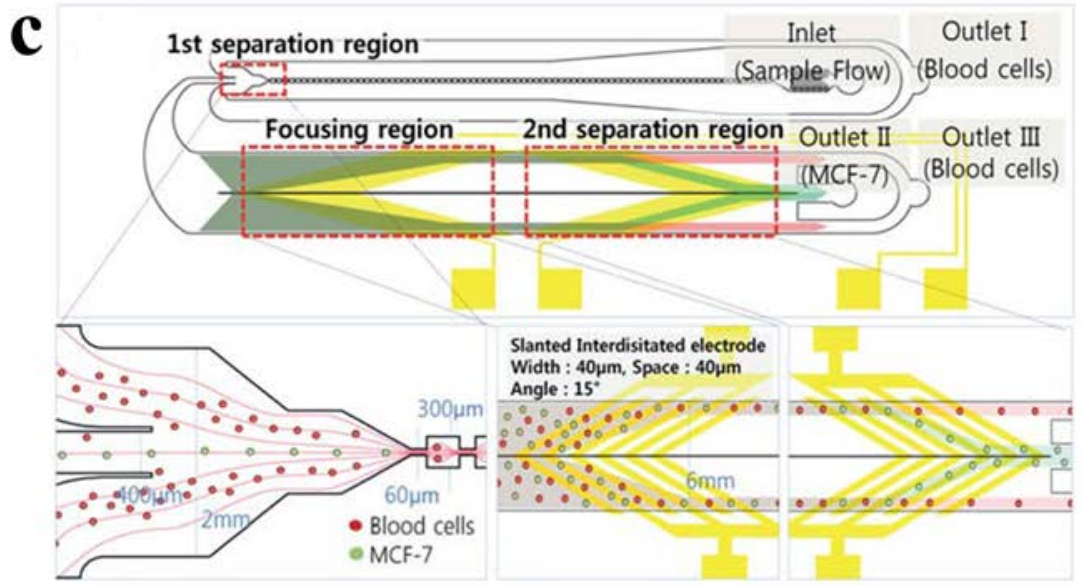
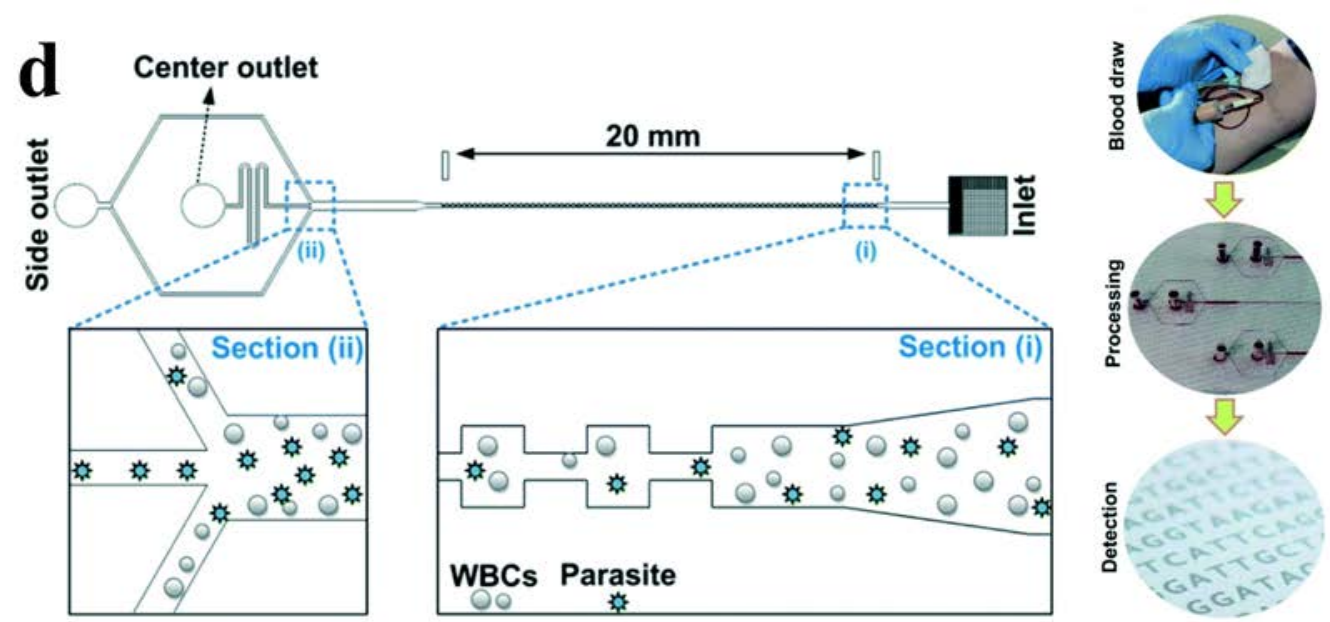

Figure 8 (a) Inertial separation of particles by size in a CEA channel under the assistance of a sheath flow. Reproduced from Ref. ${ }^{53}$ with permission from Elsevier. (b) Continuous inertial separation in a multi-orifice microchannel according to the size-dependent lateral migration termed as Multiorifice Flow Fractionation. Reproduced from Ref. ${ }^{82,83}$ with permission from the Royal Society of Chemistry and American Chemical Society Copyright (2009). (c) Continuous high-throughput separation of human breast cancer cells (MCF-7) from blood cells by a combination of multi-orifice flow fractionation (MOFF) and dielectrophoresis (DEP). Reproduced from Ref. ${ }^{85}$ with permission from the Royal Society of Chemistry. (d) Improved detection of malaria by the integration of inertial microfluidic processing and PCR detection ${ }^{86}$. 
In addition to the secondary counter-rotating flow within the cross-section of main channel around the contraction-expansion region, a horizontal micro-vortex within the contractionexpansion chamber can also be generated due to the detachment of boundary layer under a high flow speed. Shelby et al. ${ }^{87}$ was among the first to demonstrate a horizontal microvortex in a diamond shaped cavity under high flow speed in microfluidics. This microvortex can generate a rotational velocity as high as $12 \mathrm{~m} / \mathrm{s}$ and a corresponding radial acceleration in the order of $10^{6} \mathrm{~g}$. Such microvortex without any moving components are useful for the investigation of effects of high radial acceleration on biological and chemical process, as well as offering precise control and rotation of single cell ${ }^{88}$, Figure $9(\mathrm{a})$. In their work, the single cell was actively trapped and positioned at the centre of the microvortex by an optical tweezer.

Later, Di Carlo’s group ${ }^{89,90}$ introduced the concept of “Centrifuge-on-a-chip” to selectively isolate target particles/cells from heterogeneous background by the effects of inertial migration and vortex trapping. The centrifuge chip consisted of a straight channel section and symmetric expansion-contraction arrays, Figure 9(b). Rather than the optical tweezer, the selective trapping and positioning of micro-particles are based on purely hydrodynamic phenomena: the cross-stream inertial migration of micro-particles in the cavity expanding area. The mechanism of trapping has been qualitatively explained by Mach et al. ${ }^{90}$. Briefly, the selective trapping process of particles is divided into three steps: (i) inertial focusing in a straight channel, (ii) lateral migration around the expanding area, and (iii) circulation within microvortices, Figure 9(b).

The functions of trapping, enrichment, labelling and solution exchange were demonstrated in the Centrifuge chip ${ }^{90-94}$. The most significant advantage of "Centrifuge-on-a-chip" is its ability to selectively trap particles from the mainstream by size, which is believed as one of the most size-sensitive separation methods ${ }^{59}$. Di Carlo group has conducted a series of investigation on its trapping sensitivity and efficiency through a variety of bio-samples, including cancer cells spiked in blood ${ }^{90}$, pleural fluids ${ }^{92}$ and blood sample ${ }^{93}$ from cancer patients. One possible drawback of this device is that it basically works in a batch procedure, and specifically effective in trapping of rare cells (e.g. CTCs), due to limited capacity in expansion-contraction chambers. Wang et al. ${ }^{91}$ proposed a modified microvortex-aided device by adding side outlets in each chamber to continuously siphon larger particles from chambers, Figure 9(c). However, the device is still limited by the capability for bimodal separation with a single size cut-off and well-defined size difference. And it will become challenging when treating real-world samples that often includes heterogeneous mixtures of multiple particle components. Wang and Papautsky ${ }^{95}$ developed a multi-modal inertial sorter that consisted of a long straight section with series-connected separation units. The authors successfully demonstrated triple-sized particle separation with high resolution by sequencing two separation units and adjusting proper system parameters. 

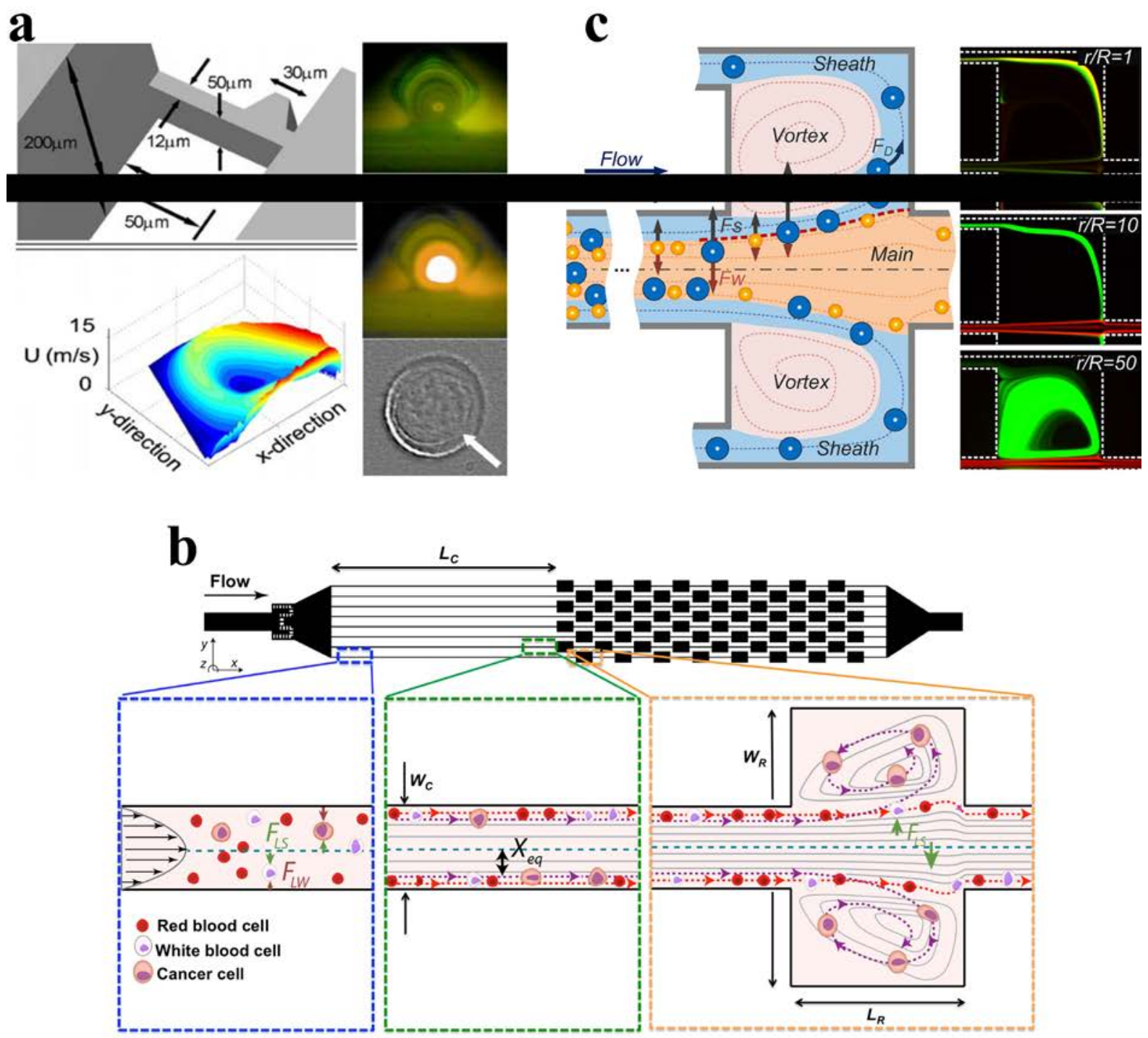

d

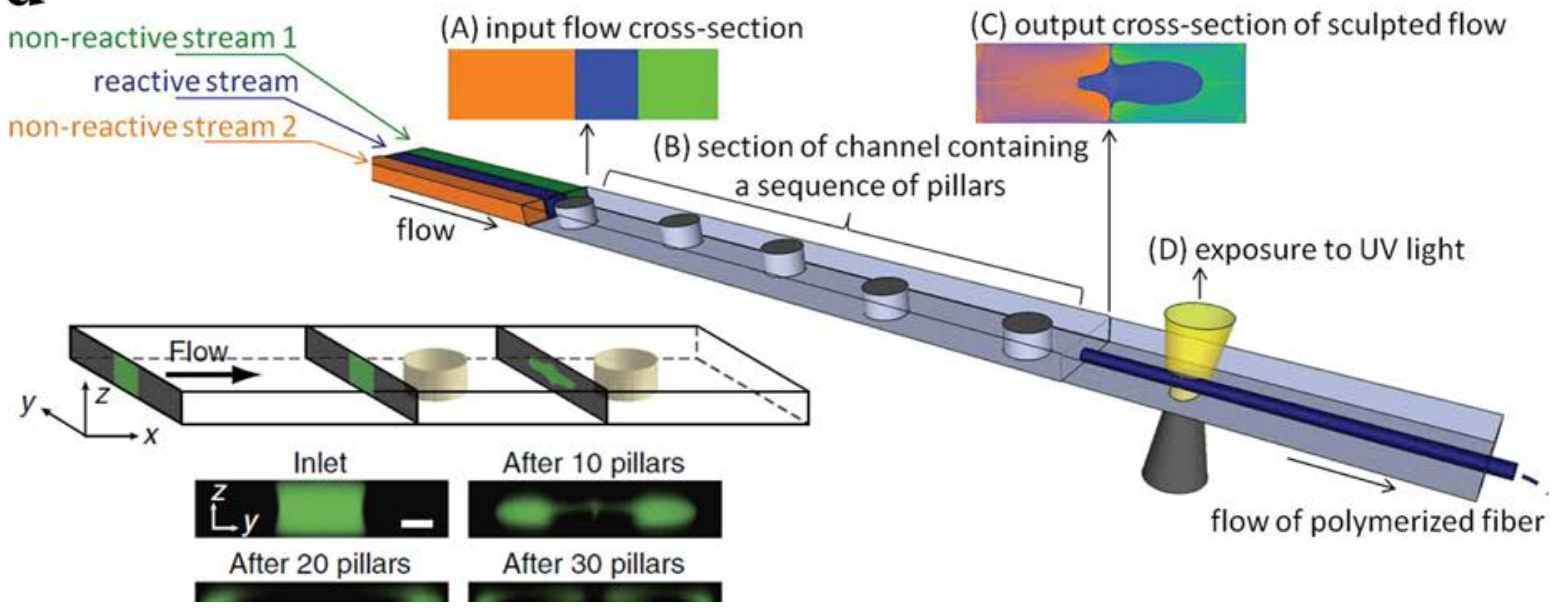

Figure 9 (a) Rotational control of single cells by microvortices in a diamond-shaped chamber. Reproduced from Ref. ${ }^{88}$ with kind permission from Springer Science \& Business Media. (b) Size-selective trapping of circulating tumor cells using "Centrifuge-on-a-chip”. Reproduced from Ref. ${ }^{93}$ with permission from the Royal Society of Chemistry. (c) Vortex-aided inertial microfluidic device for continuous sorting of particles by size. A modified side outlet in each chamber siphons the trapped large particles out continuously, meanwhile smaller particles exist from the main channel. Reproduced from Ref. ${ }^{91}$ with permission from AIP Publishing LLC. (d) 
Engineering flow deformation by programing a sequence of cylindrical pillars within a straight microchannel in inertial microfluidics and its application on synthesis of shaped polymeric microfibers. Reproduced from Ref. ${ }^{26}$,

${ }^{96}$ with permission from Nature Publishing Group and John Wiley and Sons .

Besides expansion-contraction chamber, a micro-pillar within a micro-channel will also induce irreversible twisted flows at a finite inertial flow. Furthermore, the lateral position of the pillar can be used to tune the position and shape of the net recirculating flows across the channel. It could enable precise control of fluid transformation by programming the positions of sequenced cylindrical pillars, Figure 9(d). To explore the capabilities of this sequenced pillar microchannel, Amini et al. ${ }^{26}$ have successfully demonstrated the sculpture of crosssectional shape of a laminated stream into complex geometries, moving and splitting a fluid stream, solution exchange and particle separation. Nunes et al. ${ }^{96}$ utilized this microfluidic technique to fabricate polymeric microfibers with noncircular cross-sectional shape, Figure 9(d). The computer-aided design (CAD) tool uFlow has a stored library of pre-computed fluid deformations that are produced by individual pillars in the flow channel. It is a useful tool to design the pillar sequences, and to predict the shape of net flow deformation. The cross-sectional shapes of various fabricated microfibers agreed reasonably well with that of predicted using the uFlow code.

Similar to the secondary flow induced by the contraction-expansion chamber or channel curvature, the locally induced secondary flows by sequenced pillars could also be used to modify the inertial migration progress. Chung et al. ${ }^{97}$ demonstrated single-stream focusing of microparticles through controllable cross-stream migration, which was aided by the locally tuned secondary flows from the sequential micropillars. Besides, programmable flow deformation in sequential micropillars was used to conduct solution transfer around particles or cells. The microfluidic device was applied for a functionalized bead bioassay, achieving high-yield and continuous separation of biotin-coated beads from extra FITC-biotin, as well as extraction of leukocytes from lysed blood ${ }^{98}$.

\subsection{Serpentine channel}

As we know, in spiral channels, the curvature is along a single direction, therefore secondary flow can reach steady state after a given channel length, and almost consistent within different cross-sections. Analysis of particle behaviour can be approximated by the static superposition of inertial lift force field with a secondary flow field. However, in a serpentine channel with alternating curvatures, the situation becomes more sophisticated. For example, with alternating curvatures, secondary flow may not approach steady state after each turn, the same with the movement of particles, and accumulation of this unsteady may cause unpredictable and non-intuitive behaviour of particles. Therefore, analysis of particle behaviour by the superposition method may not give a convincing explanation.

Di Carlo et al. ${ }^{43}$ investigated the effects of alternating curvatures on the particle inertial migration. In their study, original four equilibrium positions in a straight channel with square cross-section were reduced to two in a symmetric serpentine channel due to the symmetry of the system. Above a critical Dean number, focusing was perturbed. Furthermore, in an asymmetric serpentine channel, the number of equilibrium positions could be further reduced to one, focusing again became more complex as Dean number increased, Figure 10(a). From 
their understanding, the balance between inertial lift forces $\left(F_{L}\right)$ and Dean drag force $\left(F_{D}\right)$ determines the preferred location of focusing positions. Dean flow does not create particle focusing, but it acts in superposition with inertial lift forces to reduce the number of equilibrium positions created by the inertial lift forces. If $F_{D}>>F_{L}$, then no focusing will be observed, and if $F_{D}<<F_{L}$, then focusing due to inertial lift forces alone will be observed. The ratio of inertial lift force to Dean drag was scaled as: $F_{L} / F_{D} \sim \frac{2 R}{H}(a / H)^{3} R_{e}^{n},(n<0)$. This relation suggests a strong third-power dependence on the ratio of particle to channel dimensions. Even at the same Reynolds number, small particles may be still unfocused independent of channel length, because of dominant $F_{D}$, while larger particles quickly become focused. Employment of this mechanism, an inertial filtration device using an asymmetrical serpentine channel was developed and tested ${ }^{99}$. Within the expectation, large particles were well focused and small particles below a threshold remained unfocused and randomly distributed. Therefore, large particles were completely removed from the mixture, leaving behind small particles with a high purity (90\%-100\%). However, because small particles are still unfocused, plenty of them will enter the reservoirs meant for large particles, leading to unsatisfactory purity of large particles’ collection.

Inertial focusing in an asymmetric serpentine channel can produce well-defined particle focusing as well as highly regulated inter-particle spacing, which is very promising in a range of applications, e.g., the flow cytometry system ${ }^{100}$, particle active sorting ${ }^{101}$, hydrodynamic stretching of single-cells ${ }^{102}$ and bioparticle concentration ${ }^{103}$. Particle inertial focusing may replace the hydrodynamic focusing unit in the standard commercial flow cytometry, composing a compact microfluidic flow cytometry system ${ }^{100}$. Besides, it could also be integrated with ultrafast optical capturing system for automated flow-through single-cell image analysis ${ }^{104}$. For particle sorting, Toner's group ${ }^{101,105}$ developed a hybrid microfluidic device which combined deterministic lateral displacement (DLD), inertial focusing and magnetophoresis (MP) to isolate rare circulating tumour cells (CTCs) in an antigendependent and independent way, Figure 10(b). In this device, after the sample of raw blood is introduced into the chip, the blood cells first encounter an array of circular pillars with a specific gap, where DLD size-based hydrodynamic force filtrate smaller red blood cells (RBCs) from white blood cells (WBCs) and CTCs with much larger size. Subsequently, the mixture of WBCs and CTCs flows into an asymmetric serpentine microchannel where they are rapidly aligned along a single path by the inertial effects. At the end of the serpentine channel, a strong magnetic field is applied to deflect those cells that are magnetically labelled. The CTCs can then be collected separately.

Besides asymmetric serpentine channel, our group have investigated the focusing patterns and corresponding mechanisms in symmetric serpentine channels ${ }^{106-108}$. Three focusing patterns were observed by increasing the flow rate: (i) two-sided focusing, (ii) the transitional focusing, and (iii) single central focusing. From our understanding, the secondary flow due to the channel curvature, not only has mixing effects, but also can focus particles at the channel centre if the vertical component of secondary flow which is responsible for the mixing effects is suppressed by reducing the aspect ratio of channel ${ }^{106}$. After that, particles will be focused at the channel lateral centre if the secondary flow is greater than the inertial lift force. In 
contrast, if inertial lift force is stronger than the secondary flow, particles will be focused near two sidewalls. If they are in the similar order, particles will be within a transitional focusing pattern, where particles are focused as a wide streak. Employing the overlap of two-sided focusing pattern for small particles and single central focusing pattern for large particles would enable a complete separation by differential lateral equilibrium positions for binary particles ${ }^{108}$, Figure 10(c).

A DEP-inertial coupled microfluidic device was proposed through the combination of inertial lift force and DEP force ${ }^{109}$. This combination can modify the inertial focusing patterns in a serpentine channel with a vertical DEP force, which was implemented by interdigitated electrodes patterned on the bottom of microchannel. By levitating particles towards the vertical centre of channel by DEP force, the original two-sided focused particles will migrate towards the lateral centre, which indicates that increasing lateral component of secondary flow drag as well as reducing its vertical component will promote a single central focusing of the particles. This phenomenon further proves that our explanation about inertial focusing in serpentine channels is reasonable. Moreover, this device demonstrated the possibility of combining passive inertial focusing with active DEP to enable more controllability as well as maintaining the advantages of high-throughput. 

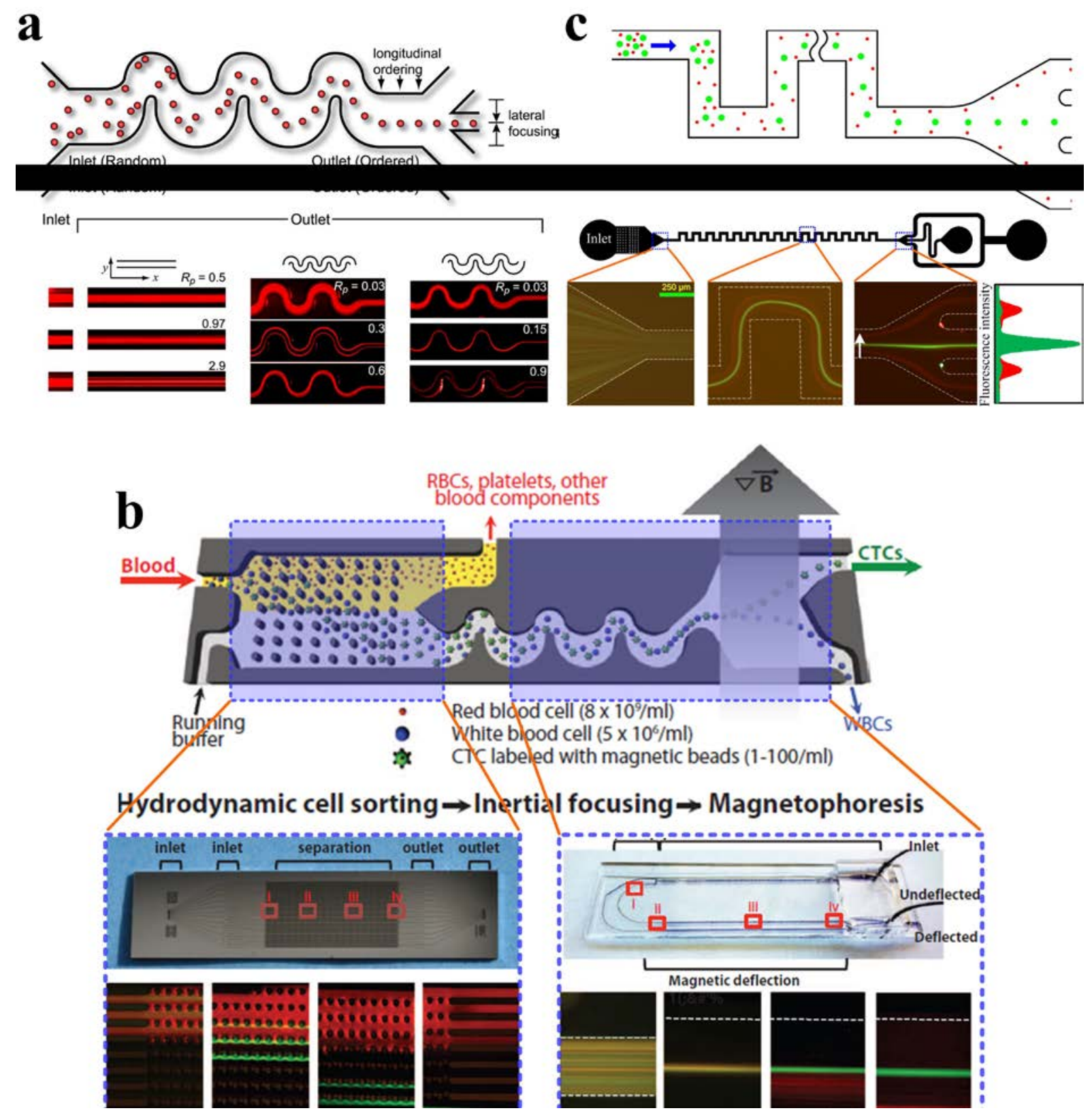

Figure 10 (a) Inertial focusing of particles in serpentine microchannels. In a straight channel with square crosssection, focusing of particles into four single streamlines is observed, with each of them facing the centre of each wall. For a symmetric serpentine channel, the symmetry of system reduces focusing to two streams. In an asymmetric serpentine channel, focusing down to a single stream is observed. Reproduced from Ref. ${ }^{43}$. Copyright (2007) National Academy of Sciences, U.S.A. (b) A hybrid microfluidic device which integrates deterministic lateral displacement (DLD), inertial focusing in asymmetrical serpentine channel and magnetophoresis (MP) to isolate rare circulating tumour cells (CTCs) in an antigen-dependent and independent way. Reproduced from Ref. ${ }^{101}$ with permission from the American Association for the Advancement of Science. (c) Size-based separation of binary particles by their differential equilibrium positions in a symmetric serpentine microchannel. Reproduced from Ref. ${ }^{108}$ with permission from Nature Publishing Group.

\section{CONCLUSIONS AND OUTLOOK}

In this review, we first discussed the fundamental dynamics of particle movement. Several lateral forces might act on particles in an inertial microfluidic device. Viscous drag force, arising from the velocity difference between fluid element and particles, and basically retains 
the particles within fluid streamlines. Lateral forces, such as Magnus force, Saffman force, shear gradient lift force and wall lift force are perpendicular to the main streamlines, and account for the intriguing phenomenon of particle inertial migration. In a wall-bounded Poiseuille flow, Magnus and Saffman force are normally neglected. And the balance of centre-directed wall lift force and wall-directed shear gradient lift force can well explain the experimental observation by Segre and Silberberg ${ }^{12,13,17}$. Besides, the properties of particles (e.g. deformability, shape, size) and the fluid property (e.g. viscoelasticity) may also influence the lateral migration of particles $45,49,110$.

Subsequently, a comprehensive review of recent progress of the inertial microfluidic technology was presented, according to the structure of functional microchannels, i.e. straight 42, 44, 49, 54, straight channel with pillar arrays ${ }^{26,97,111}$ or expansion-contraction arrays ${ }^{25,79,82}$, spiral $^{77,112,113}$, and serpentine ${ }^{43,106,114}$. These inertial microfluidic devices have been widely explored for their applications in biomedicine and industry, such as extraction of blood plasma $^{80,107}$, separation of particles and cells ${ }^{53,68,72,108,115}$, solution exchange ${ }^{98,116,117}$, cell enrichment ${ }^{118}$, isolation of circulating tumour cells (CTCs) ${ }^{69,71,75,81,90,92,93,101}$, detection of malaria pathogen ${ }^{86,119}$, microfiber fabrication ${ }^{96}$, cell cycle synchronization ${ }^{73}$, cell encapsulation ${ }^{65}$, and hydrodynamic stretching of single cell ${ }^{102,120}$ etc. There are also several commercial/quasi-commercial prototypes reported based on the inertial microfluidic techniques ${ }^{35}$, e.g. CTC-iChip by Johnson \& Johnson ${ }^{101}$, ClearCell FX system by Clearbridge BioMedics ${ }^{75,121}$, mechanophenotyping platform by CytoVale ${ }^{122}$, as well as Microfluidic “centrifuge-on-a-chip” by Vortex Biosciences ${ }^{90,123}$.

Although there have been extensive investigations on the mechanism of particle inertial focusing, and a variety of applications on particle/cell separation have been demonstrated, quantitative design rules are still lacking for particle inertial manipulation in different shaped channels. Therefore, more dedicated work is needed to uncover the detailed underlying mechanism, and provide explicit rules to the end users even without specialized knowledge and experience about inertial microfluidics. In addition, more efforts are needed to improve the separation resolution and processing speed through optimization of channel structure and

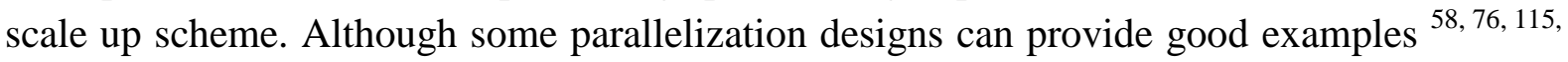
${ }^{124}$, a general design guidelines and optimization scheme based on different channel structure would be more helpful.

Combination of passive inertial microfluidics with active manipulation techniques will enable more versatile and powerful functionality. Several manipulation techniques may be connected in series with independent physics, or in parallel with coupled physics. Ozkumur et al. ${ }^{101,105}$ reported an integrated microfluidic device which combined deterministic lateral displacement (DLD), inertial focusing and magnetophoresis (MP) to isolate rare circulating tumour cells (CTCs) in an antigen-dependent and independent way. Meanwhile, Moon et al. ${ }^{85}$ successfully separated human breast cancer cells (MCF-7) from spiked blood sample by a combination of multi-orifice flow fractionation (MOFF) and dielectrophoresis (DEP) techniques. In both works, although the physics in each section is common and independent, actually they are weakly linked, because the interface between each section, such as flow rate or pressure, needs to be balanced carefully, so that each section can work normally. 
We proposed a DEP-inertial microfluidic device, where negative DEP force and inertial lift force are coupled along the vertical direction to adjust the focusing positions of the particles in three dimensions ${ }^{109}$. Based on this platform, the DEP force could be used as a force probe to measure the magnitude of inertial lift force. Besides the DEP force, magnetic, acoustic or other active forces may also be combined with inertial lift force in inertial microfluidics, to achieve more versatility and flexibility for Microfluidic system.

Current study on particle manipulation by inertial microfluidics is often limited for particles within micro-scale region. The territory of nano-scale particles (DNA, protein and virus etc.) has not been explored yet in inertial microfluidics. For nano-scale particles, the Brownian motion becomes more obvious, and maybe even overcomes its inertial effects. Therefore, a reduced channel size (sub-micro to nano-scale) may be not a necessary consequence, and introduction of additional viscoelastic effects could be a possible alternative ${ }^{125}$.

To date, inertial focusing has been conducted where particles are suspended within liquid phrase. There is no reported work for particle-air system, i.e. aerosol. No matter from the aspects of fundamental exploration on particle movement within micro-airway, or from the aspects of its potential application in biomedicine and industry, e.g. understanding functionality of respiratory system, inhalation drug delivery and air pollution purification etc., this kind of research is still very meaningful.

In conclusion, although significant progress has been achieved for inertial microfluidics over the last decades, even with a few commercial prototypes developed, inertial microfluidics itself is still in its early development stage. A wide region of its raw territory has been waiting for exploration and exploitation. We believe that in the near future inertial microfluidics remains a hot research topic with expanding applications, because of the superior advantages of the technology, such as high throughput, simple structure and low cost.

\section{ACKNOWLEDGEMENTS}

This work was partially supported by the University of Wollongong through a UIC grant and China Scholarship Council.

\section{REFERENCES}

$1 \quad$ G. M. Whitesides, Nature, 2006, 442, 368-373.

2 E. K. Sackmann, A. L. Fulton and D. J. Beebe, Nature, 2014, 507, 181-189.

3 A. A. S. Bhagat, H. Bow, H. W. Hou, S. J. Tan, J. Han and C. T. Lim, Med. Biol. Eng. Comput., 2010, 48, 999-1014.

$4 \quad$ D. Di Carlo, Lab Chip, 2009, 9, 3038-3046.

5 A. J. Mach, O. B. Adeyiga and D. Di Carlo, Lab Chip, 2013, 13, 1011-1026.

$6 \quad$ B. Çetin and D. Li, Electrophoresis, 2011, 32, 2410-2427.

$7 \quad$ T. P. Forbes and S. P. Forry, Lab Chip, 2012, 12, 1471-1479.

8 Z. Wang and J. Zhe, Lab Chip, 2011, 11, 1280-1285.

9 D. G. Grier, Nature, 2003, 424, 810-816.

10 M. Yamada, M. Nakashima and M. Seki, Anal. Chem., 2004, 76, 5465-5471.

11 L. R. Huang, E. C. Cox, R. H. Austin and J. C. Sturm, Science, 2004, 304, 987-990.

12 G. Segre, Nature, 1961, 189, 209-210. 
14 J. B. McLaughlin, J. Fluid Mech., 1993, 246, 249-265.

15 D. D. JOSEPH and D. OCANDO, J. Fluid Mech., 2002, 454, 263-286.

16 P. Cherukat and J. B. Mclaughlin, J. Fluid Mech., 1994, 263, 1-18.

17 E. S. ASMOLOV, J. Fluid Mech., 1999, 381, 63-87.

18 B. Chun and A. Ladd, Phys. Fluids, 2006, 18, 031704.

19 T. Tanaka, T. Ishikawa, K. Numayama-Tsuruta, Y. Imai, H. Ueno, T. Yoshimoto, N. Matsuki and T. Yamaguchi, Biomed. Microdevices, 2012, 14, 25-33.

20 B. H. YANG, J. WANG, D. D. JOSEPH, H. H. HU, T.-W. PAN and R. GLOWINSKI, J. Fluid Mech., 2005, 540, 109-131.

21 K. Hood, S. Lee and M. Roper, J. Fluid Mech., 2015, 765, 452-479.

22 J.-P. Matas, J. F. Morris and É. Guazzelli, J. Fluid Mech., 2004, 515, 171-195.

23 S. Berger, L. Talbot and L. Yao, Annu. Rev. Fluid Mech., 1983, 15, 461-512.

24 M. G. Lee, S. Choi and J. K. Park, Appl. Phys. Lett., 2009, 95, 051902.

25 J. Zhang, M. Li, W. Li and G. Alici, J. Micromech. Microeng., 2013, 23, 085023.

26 H. Amini, E. Sollier, M. Masaeli, Y. Xie, B. Ganapathysubramanian, H. A. Stone and D. Di Carlo, Nat. Commun., 2013, 4, 1826.

27 J. F. Richardson, J. M. Coulson, J. Harker and J. Backhurst, Chemical Engineering: Particle technology and separation processes, Butterworth-Heinemann, 2002.

28 R. M. Mazo, Brownian motion: fluctuations, dynamics, and applications, Clarendon press Oxford, 2002.

29 R. Clift, J. R. Grace and M. E. Weber, Bubbles, drops, and particles, Courier Corporation, 2005.

30 E. Michaelides, Particles, bubbles \& drops: their motion, heat and mass transfer, World Scientific, Singapore, 2006.

31 S. Rubinow and J. B. Keller, J. Fluid Mech., 1961, 11, 447-459.

32 J. Matas, J. Morris and E. Guazzelli, Oil Gas Sci. Technol., 2004, 59, 59-70.

33 J. Feng, H. H. Hu and D. D. Joseph, J. Fluid Mech., 1994, 277, 271-301.

34 P. G. Saffman, J. Fluid Mech., 1965, 22, 385-400.

35 H. Amini, W. Lee and D. Di Carlo, Lab Chip, 2014, 14, 2739-2761.

$36 \quad$ Y. W. Kim and J. Y. Yoo, Lab Chip, 2009, 9, 1043-1045.

37 H. Brenner, Chem. Eng. Sci., 1961, 16, 242-251.

38 R. Cox and S. Hsu, Int. J. Multiphase Flow, 1977, 3, 201-222.

39 P. Vasseur and R. G. Cox, J. Fluid Mech., 1977, 80, 561-591.

$40 \quad$ P. Cherukat and J. McLaughlin, Int. J. Multiphase Flow, 1990, 16, 899-907.

41 A. A. S. Bhagat, S. S. Kuntaegowdanahalli and I. Papautsky, Microfluid. Nanofluid., 2009, 7, 217-226.

42 J. Zhou and I. Papautsky, Lab Chip, 2013, 13, 1121-1132.

43 D. Di Carlo, D. Irimia, R. G. Tompkins and M. Toner, Proc. Natl. Acad. Sci. U.S.A., 2007, 104, 18892-18897.

44 D. Di Carlo, J. F. Edd, K. J. Humphry, H. A. Stone and M. Toner, Phys. Rev. Lett., 2009, 102, 94503.

45 S. C. Hur, N. K. Henderson-MacLennan, E. R. McCabe and D. Di Carlo, Lab Chip, 2011, 11, 912-920.

46 C. A. Stan, A. K. Ellerbee, L. Guglielmini, H. A. Stone and G. M. Whitesides, Lab Chip, 2013, 13, 365-376.

$47 \quad$ P.-H. Chan and L. Leal, J. Fluid Mech., 1979, 92, 131-170.

48 H. W. Hou, A. A. S. Bhagat, A. G. L. Chong, P. Mao, K. S. W. Tan, J. Han and C. T. Lim, Lab Chip, 2010, 10, 2605-2613.

49 S. C. Hur, S.-E. Choi, S. Kwon and D. Di Carlo, Appl. Phys. Lett., 2011, 99, 044101. 

McKinley and M. Toner, Nat. Commun., 2014, 5, Art NO. 4120.

51 Y.-S. Choi, K.-W. Seo and S.-J. Lee, Lab Chip, 2011, 11, 460-465.

52 A. J. Chung, D. R. Gossett and D. Di Carlo, Small, 2013, 9, 685-690.

M. G. Lee, S. Choi and J. K. Park, J. Chromatogr. A, 2011, 1218, 4138-4143.

H. Amini, E. Sollier, W. M. Weaver and D. Di Carlo, Proc. Natl. Acad. Sci. U.S.A., 2012, 109, 11593-11598.

55 W. Lee, H. Amini, H. A. Stone and D. Di Carlo, Proc. Natl. Acad. Sci. U.S.A., 2010, 107, 22413-22418.

56 C. Liu, G. Hu, X. Jiang and J. Sun, Lab Chip, 2015, 15, 1168-1177.

57 S. C. Hur, T. Z. Brinckerhoff, C. M. Walthers, J. C. Dunn and D. Di Carlo, PLoS One, 2012, 7, e46550.

$58 \quad$ A. J. Mach and D. Di Carlo, Biotechnol. Bioeng., 2010, 107, 302-311.

59 J. Zhou, P. V. Giridhar, S. Kasper and I. Papautsky, Lab Chip, 2013, 13, 1919-1929.

60 J. S. Dudani, D. E. Go, D. R. Gossett, A. P. Tan and D. Di Carlo, Anal. Chem., 2014, 86, $1502-1510$.

61 A. P. Tan, J. S. Dudani, A. Arshi, R. J. Lee, T. Henry, D. R. Gossett and D. Di Carlo, Lab Chip, 2014, 14, 522-531.

62 S. C. Hur, H. T. K. Tse and D. Di Carlo, Lab Chip, 2010, 10, 274-280.

63 T. M. Squires and S. R. Quake, Rev. Mod. Phys., 2005, 77, 977.

64 A. A. S. Bhagat, S. S. Kuntaegowdanahalli, N. Kaval, C. J. Seliskar and I. Papautsky, Biomed. Microdevices, 2010, 12, 187-195.

65 E. W. Kemna, R. M. Schoeman, F. Wolbers, I. Vermes, D. A. Weitz and A. van den Berg, Lab Chip, 2012, 12, 2881-2887.

66 D. H. Yoon, J. B. Ha, Y. K. Bahk, T. Arakawa, S. Shoji and J. S. Go, Lab Chip, 2008, 9, 87-90.

67 A. A. S. Bhagat, S. S. Kuntaegowdanahalli and I. Papautsky, Lab Chip, 2008, 8, 1906-1914.

68 S. S. Kuntaegowdanahalli, A. A. S. Bhagat, G. Kumar and I. Papautsky, Lab Chip, 2009, 9, 2973-2980.

69 J. Sun, M. Li, C. Liu, Y. Zhang, D. Liu, W. Liu, G. Hu and X. Jiang, Lab Chip, 2012, 12, 3952-3960.

70 J. Sun, C. Liu, M. Li, J. Wang, Y. Xianyu, G. Hu and X. Jiang, Biomicrofluid, 2013, 7, 011802.

71 M. E. Warkiani, G. Guan, K. B. Luan, W. C. Lee, A. A. S. Bhagat, P. K. Chaudhuri, D. S.-W. Tan, W. T. Lim, S. C. Lee and P. C. Chen, Lab Chip, 2014, 14, 128-137.

72 L. Wu, G. Guan, H. W. Hou, A. A. S. Bhagat and J. Han, Anal. Chem., 2012, 84, 9324-9331.

73 W. C. Lee, A. A. S. Bhagat, S. Huang, K. J. Van Vliet, J. Han and C. T. Lim, Lab Chip, 2011, 11, 1359-1367.

74 W. Sheng, T. Chen, R. Kamath, X. Xiong, W. Tan and Z. H. Fan, Anal. Chem., 2012, 84, 4199-4206.

75 H. W. Hou, M. E. Warkiani, B. L. Khoo, Z. R. Li, R. A. Soo, D. S.-W. Tan, W.-T. Lim, J. Han, A. A. S. Bhagat and C. T. Lim, Sci. Rep., 2013, 3, Art NO. 1259.

76 M. E. Warkiani, B. L. Khoo, D. S.-W. Tan, A. A. S. Bhagat, W.-T. Lim, Y. S. Yap, S. C. Lee, R. A. Soo, J. Han and C. T. Lim, Analyst, 2014, 139, 3245-3255.

77 G. Guan, L. Wu, A. A. Bhagat, Z. Li, P. C. Chen, S. Chao, C. J. Ong and J. Han, Sci. Rep., 2013, 3, Art NO. 1475.

78 B. L. Khoo, M. E. Warkiani, D. S.-W. Tan, A. A. S. Bhagat, D. Irwin, D. P. Lau, A. S. Lim, K. H. Lim, S. S. Krisna and W.-T. Lim, PLoS One, 2014, 9, e99409. 
80 M. G. Lee, S. Choi, H. J. Kim, H. K. Lim, J. H. Kim, N. Huh and J. K. Park, Appl. Phys. Lett., 2011, 98, 253702.

81 M. G. Lee, J. H. Shin, C. Y. Bae, S. Choi and J.-K. Park, Anal. Chem., 2013, 85, 6213-6218.

82 J. S. Park, S. H. Song and H. I. Jung, Lab Chip, 2009, 9, 939-948.

83 J.-S. Park and H.-I. Jung, Anal. Chem., 2009, 81, 8280-8288.

84 T. S. Sim, K. Kwon, J. C. Park, J.-G. Lee and H.-I. Jung, Lab Chip, 2011, 11, 93-99.

85 H. S. Moon, K. Kwon, S. I. Kim, H. Han, J. Sohn, S. Lee and H. I. Jung, Lab Chip, 2011, 11, 1118-1125.

86 M. E. Warkiani, A. K. P. Tay, B. L. Khoo, X. Xiaofeng, J. Han and C. T. Lim, Lab Chip, 2015, 15, 1101-1109.

87 J. P. Shelby, D. S. Lim, J. S. Kuo and D. T. Chiu, Nature, 2003, 425, 38-38.

88 D. T. Chiu, Anal. Bioanal. Chem., 2007, 387, 17-20.

89 S. C. Hur, A. J. Mach and D. Di Carlo, Biomicrofluid, 2011, 5, 022206.

90 A. J. Mach, J. H. Kim, A. Arshi, S. C. Hur and D. Di Carlo, Lab Chip, 2011, 11, 2827-2834.

91 X. Wang, J. Zhou and I. Papautsky, Biomicrofluid, 2013, 7, 044119.

92 J. Che, A. J. Mach, D. E. Go, I. Talati, Y. Ying, J. Rao, R. P. Kulkarni and D. Di Carlo, PLoS One, 2013, 8, e78194.

93 E. Sollier, D. E. Go, J. Che, D. R. Gossett, S. O'Byrne, W. M. Weaver, N. Kummer, M. Rettig, J. Goldman and N. Nickols, Lab Chip, 2014, 14, 63-77.

94 J. Zhou, S. Kasper and I. Papautsky, Microfluid. Nanofluid., 2013, 15, 611-623.

95 X. Wang and I. Papautsky, Lab Chip, 2015, 15, 1350-1359.

96 J. K. Nunes, C. Y. Wu, H. Amini, K. Owsley, D. Di Carlo and H. A. Stone, Adv. Mater., 2014, 26, 3712-3717.

97 A. J. Chung, D. Pulido, J. C. Oka, H. Amini, M. Masaeli and D. Di Carlo, Lab Chip, 2013, 13, 2942-2949.

98 E. Sollier, H. Amini, D. Go, P. Sandoz, K. Owsley and D. Di Carlo, Microfluid. Nanofluid., 2015, 1-13.

99 D. Di Carlo, F. Jon, D. Irimia, R. G. Tompkins and M. Toner, Anal. Chem., 2008, 80, 2204-2211.

100 J. Oakey, R. W. Applegate Jr, E. Arellano, D. D. Carlo, S. W. Graves and M. Toner, Anal. Chem., 2010, 82, 3862-3867.

101 E. Ozkumur, A. M. Shah, J. C. Ciciliano, B. L. Emmink, D. T. Miyamoto, E. Brachtel, M. Yu, P.-i. Chen, B. Morgan and J. Trautwein, Sci. Transl. Med., 2013, 5, 179 ra147.

102 D. R. Gossett, T. Henry, S. A. Lee, Y. Ying, A. G. Lindgren, O. O. Yang, J. Rao, A. T. Clark and D. Di Carlo, Proc. Natl. Acad. Sci. U.S.A., 2012, 109, 7630-7635.

103 J. M. Martel, K. C. Smith, M. Dlamini, K. Pletcher, J. Yang, M. Karabacak, D. A. Haber, R. Kapur and M. Toner, Sci. Rep., 2015, 5, Art NO. 11300.

104 K. Goda, A. Ayazi, D. R. Gossett, J. Sadasivam, C. K. Lonappan, E. Sollier, A. M. Fard, S. C. Hur, J. Adam and C. Murray, Proc. Natl. Acad. Sci. U.S.A., 2012, 109, 11630-11635.

105 N. M. Karabacak, P. S. Spuhler, F. Fachin, E. J. Lim, V. Pai, E. Ozkumur, J. M. Martel, N. Kojic, K. Smith and P.-i. Chen, Nat. Protoc., 2014, 9, 694-710.

106 J. Zhang, W. Li, M. Li, G. Alici and N.-T. Nguyen, Microfluid. Nanofluid., 2013, 17, 305-316.

107 J. Zhang, S. Yan, W. Li, G. Alici and N.-T. Nguyen, RSC Adv., 2014, 4, 3314933159. 
108 J. Zhang, S. Yan, R. Sluyter, W. Li, G. Alici and N.-T. Nguyen, Sci. Rep., 2014, 4, Art No. 4527.

109 J. Zhang, S. Yan, G. Alici, N.-T. Nguyen, D. Di Carlo and W. Li, RSC Adv., 2014, 4, 62076-62085.

110 F. Del Giudice, G. Romeo, G. D'Avino, F. Greco, P. A. Netti and P. L. Maffettone, Lab Chip, 2013, 13, 4263-4271.

111 D. Stoecklein, C.-Y. Wu, K. Owsley, Y. Xie, D. Di Carlo and B. Ganapathysubramanian, Lab Chip, 2014, 14, 4197-4204.

112 N. Xiang, H. Yi, K. Chen, D. Sun, D. Jiang, Q. Dai and Z. Ni, Biomicrofluid, 2013, 7, 044116.

113 J. M. Martel and M. Toner, Sci. Rep., 2013, 3, Art NO. 3340.

114 D. R. Gossett and D. D. Carlo, Anal. Chem., 2009, 81, 8459-8465.

115 M. E. Warkiani, A. K. P. Tay, G. Guan and J. Han, Sci. Rep., 2015, 5, Art NO. 11018

116 J. S. Dudani, D. R. Gossett, T. Henry, R. J. Lamm, R. P. Kulkarni and D. Di Carlo, Biomicrofluid, 2015, 9, 014112.

117 D. R. Gossett, H. T. K. Tse, J. S. Dudani, K. Goda, T. A. Woods, S. W. Graves and D. Di Carlo, Small, 2012, 8, 2757-2764.

118 J. M. Martel, K. C. Smith, M. Dlamini, K. Pletcher, J. Yang, M. Karabacak, D. A. Haber, R. Kapur and M. Toner, Sci. Rep., 2015, 5, Art NO. 11300.

119 C. M. Birch, H. W. Hou, J. Han and J. C. Niles, Sci. Rep., 2015, 5, Art NO. 11347.

120 J. S. Dudani, D. R. Gossett, T. Henry and D. Di Carlo, Lab Chip, 2013, 13, 37283734.

121 C. BioMedics, Clearcell FX System, http://www.clearbridgebiomedics.com/, Accessed 28th Oct, 2015.

122 T. Henry, D. R. Gossett, Y. S. Moon, M. Masaeli, M. Sohsman, Y. Ying, K. Mislick, R. P. Adams, J. Rao and D. Di Carlo, Sci. Transl. Med., 2013, 5, 212 ra163.

123 V. Biosciences, Vortex technology/microfluidic "centrifuge-on-a-chip", http://www.vortexbiosciences.com/, Accessed 28th Oct, 2015.

124 J. Hansson, J. M. Karlsson, T. Haraldsson, H. Brismar, W. van der Wijngaart and A. Russom, Lab Chip, 2012, 12, 4644-4650.

125 J. Y. Kim, S. W. Ahn, S. S. Lee and J. M. Kim, Lab Chip, 2012, 12, 2807-2814. 